\title{
Blessings in disguise: a review of phytochemical composition and antimicrobial activity of plants belonging to the genus Eryngium
}

Sinem Aslan Erdem ${ }^{1}$, Seyed Fazel Nabavi ${ }^{2}$, Ilkay Erdogan Orhan ${ }^{3}$, Maria Daglia ${ }^{4}$, Morteza Izadi ${ }^{5}$

and Seyed Mohammad Nabavi $i^{*}$

\begin{abstract}
Medicinal and edible plants play a crucial role in the prevention and/or mitigation of different human diseases from ancient times to today. In folk medicine, there are different plants used for infectious disease treatment. During the past two decades, much attention has been paid to plants as novel alternative therapeutic agents for the treatment of infectious diseases due to their bioactive natural compounds such as phenol, flavonoids, tannins, etc. The genus Eryngium (Apiaceae) contains more than 250 flowering plant species, which are commonly used as edible and medicinal plants in different countries. In fact, some genus Eryngium species are used as spices and are cultivated throughout the world and others species are used for the treatment of hypertension, gastrointestinal problems, asthma, burns, fevers, diarrhea, malaria, etc. Phytochemical analysis has shown that genus Eryngium species are a rich source of flavonoids, tannins, saponins, and triterpenoids. Moreover, eryngial, one the most important and major compounds of genus Eryngium plant essential oil, possesses a significant antibacterial effect. Thus, the objective of this review is to critically review the scientific literature on the phytochemical composition and antibacterial effects of the genus Eryngium plants. In addition, we provide some information about traditional uses, cultivation, as well as phytochemistry.
\end{abstract}

Keywords: Antibacterial, Eryngial, Eryngium, Flavonoids, Saponins, Infection

\section{Background}

Infectious diseases are known as one of the most important leading causes of long and short-term morbidity and mortality worldwide $[1,2]$. According to the World Health Organization, in 2011, infectious diseases were responsible for approximately 18 million deaths worldwide. In addition to the high prevalence of infectious diseases, there are some microorganisms resistant to antibiotic therapy, which lead to the increase of death rate due their ability to acquire and transmit drug resistance [2]. Nowadays, antibiotic resistance is known as one the most important and challenging health problems in the global health programs. Therefore, during the past two decades, much attention has been paid to

\footnotetext{
* Correspondence: Nabavi208@gmail.com

${ }^{2}$ Applied Biotechnology Research Center, Baqiyatallah University of Medical Sciences, P.O. Box 19395-5487, Tehran, Iran

Full list of author information is available at the end of the article
}

the discovery and development of natural multi-target antimicrobial agents with high efficacy and low adverse effects [3]. Natural products are known as one of the most important and effective drugs for human disease treatment [4-6]. In addition to their efficacy, natural products are mostly non-toxic and therefore, they can be used as safe therapeutic strategies [7-9]. A plethora of scientific evidence reported that edible and medicinal plants have significant potential to synthesize antimicrobial agents as their defense mechanisms against biotic stresses such as microorganisms [10]. It has been reported that plant-derived antimicrobial compounds can be categorized into the different groups such as phenols, flavonoids, terpenoids, lectins, polypeptides, polyacetylenes as well as alkaloids [11]. In addition, in traditional medicine, many edible and medicinal plants have been widely used for the treatment of different infectious diseases [11, 12]. 
The genus Eryngium contains more than 250 flowering species worldwide [13]. Genus Eryngium is the largest and most complex genus in Apiaceae family [13, 14]. Some species in the genus Eryngium are endangered such as $E$. alpinum L., E. aristulatum Jeps., E. constancei M.Y. Sheikh, E. cuneifolium Small, and E. viviparum J. Gay [13, 14]. According to the morphological studies, the genus Eryngium has been classified into five subgenera, including Eryngium subgenus Eryngium, E. subgenus Monocotyloidea, E. subgenus Fruticosa, E. subgenus Semiaquatica, and E. subgenus Foetida [15, 16]. E. subgenus Eryngium is the most common one throughout Europe, Africa and Asia, while the other subgenera are widely distributed in Australia $[15,16]$. However, infrageneric analysis through sequence data of chloroplast DNA trnQ-trnK 5'-exon and nuclear ribosomal DNA ITS regions showed that there are two different subgenera including Eryngium and Monocotyloidea [17-19].

Like the other members of Apiaceae family, the genus Eryngium plants have various culinary and/or medicinal uses (Fig. 1) [20-23]. Several species have been widely used in traditional medicine such as E. foetidum L., $E$. caucasicum Trautv. (syn. Eryngium caeruleum M. Bieb.), E. maritimum L., E. planum L., E. dichotomum Desf., E. campestre L. and E. creticum Lam. [24-27], whereas E. foetidum and E. caucasicum have been widely cultivated in some Asian countries such as Iran, Turkey, etc. [27, 28].
E. caucasicum is known as one the most common edible leafy vegetables in northern part of Iran and widely used in different foodstuff, pickles, etc. [28, 29]. Besides, the fruits of $E$. foetidum are known as common edible food components in Nigeria [30]. Actually, some Eryngium species are also used as ornamental plants [31]. A mountain of scientific evidence has shown that different species of the genus Eryngium possess antimicrobial effects under in vitro and in vivo conditions [32-36].

Therefore, the present paper aims to review the scientific literature on the phytochemical composition and antimicrobial activities of essential oils and extracts obtained from genus Eryngium species. In addition, we discuss about traditional uses, cultivation and phytochemistry of Eryngium species to provide a complete picture of this genus.

\section{Materials and methods}

Data sources and search strategy

Data were collected from Medline, Pubmed, Scopus, Web of Science (ISI Web of Knowledge), Science Direct, Embase, and BIOSIS Previews (from 1950 to July 20, 2015), via searching of these keywords: "Eryngium and phytochemistry or chemical compounds", "Eryngium and antibacterial", "Eryngium and antimicrobial", and "Eryngium and biological effect". We also scanned the reference list of each paper and searched Cochrane review library.

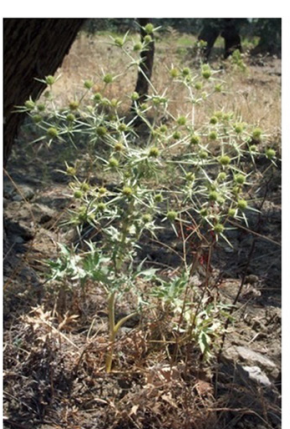

A

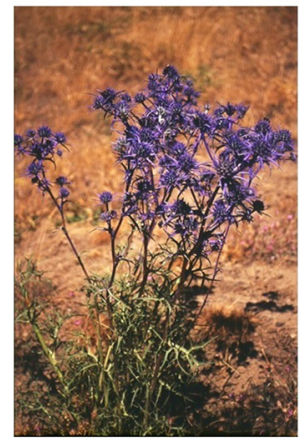

E

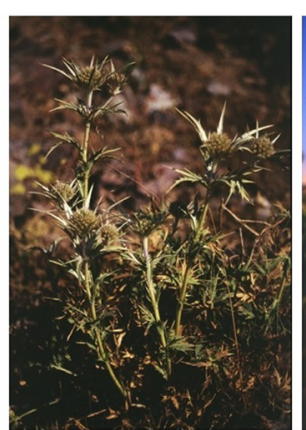

B

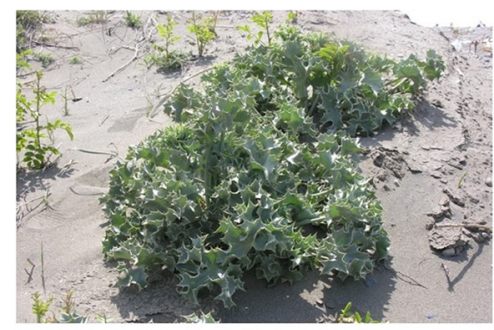

$\mathrm{F}$

$\mathrm{C}$
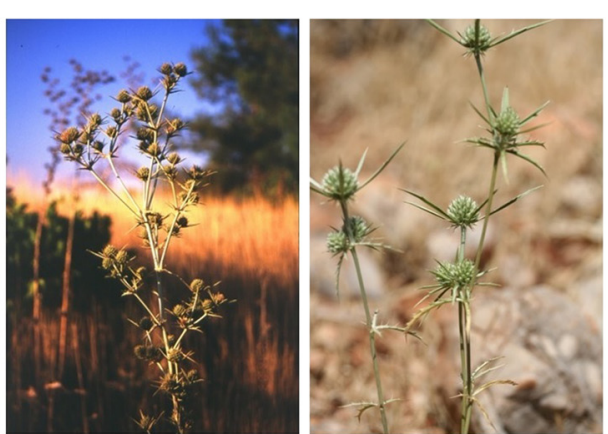

D

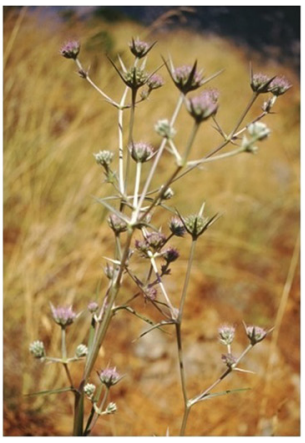

G

Fig. 1 Some of species from Enyngium genus, Enyngium campestre L. (a), Enyngium davisii Kit Tan \& Yildiz (b), Enyngium isauricum Contandr. \& Quezel (c), Enyngium falcatum Delar. (d), Enyngium kotschyi Boiss. (e), Enyngium maritimum L. (f), Enyngium trisectum Wörz \& H. Duman. (g) 
Thereafter, the bibliographies of collected data were screened for further publications. Finally, collected data were analyzed and judged by second and third authors according to the scientific standard of conduct. However, some of the references may be published after the initial search date July 2015.

\section{Traditional uses}

In traditional medicine, some of Eryngium species have been used for treatment of several human diseases [24-27]. It has been reported that E. campestre L. is widely used as antitussive, stimulant, aphrodisiac, and diuretic agent in Turkish traditional medicine $[37,38]$. E. caucasicum is one of the most important edible plants in the northern part of Iran due to its multiple beneficial effects on human health $[29,31,39,40]$. Moreover, E. creticum has been used as hypoglycemic plant in the Jordanian traditional medicine [41], while E. elegans Cham. \& Schltdl. has been reported to be utilized as diuretic agent in the Argentinian traditional medicine [42]. Most of Eryngium species are also known as medicinal plants worldwide for the treatment of several human diseases such as diarrhea, gastrointestinal problems, bladder and kidney dysfunctions, and venereal diseases [24-27, 43-45]. In Chinese traditional medicine, E. foetidum is widely used for treatment of inflammation [24] and E. yuccifolium Michx. roots have been traditionally used to treat snakebites, toothache $[27,46,47]$, digestive problems, diarrhea, headache, etc. [24-27, 43-45]. A large scale of evidence has pointed out to multiple pharmacological effects of the genus Eryngium species including antioxidant, anti-inflammatory, antihemolytic, antinociceptive effects, and protective agent against neurodegenerative deseases $[25,28,48-50]$. For instance, Eryngium planum, which is a rare medicinal plant, was studied to determine the effect of subchronic administration of a $70 \%$ ethanol root extract $(200 \mathrm{mg} / \mathrm{kg}$, p.o.) on behavioral and cognitive responses in experimental animals (Wistar rats) linked with the expression levels of mRNA coding for enzymes such as acetylcholinesterase (AChE), butyrylcholinesterase (BuChE), and beta-secretase (BACE-1). At the end of the experiment, after the last dose of the Eryngium extract, scopolamine (SC) was administered intraperitoneally to a group of animals (treated). In the animals treated with the Eryngium extract, an improvement in long-term memory produced by the EP extract in both scopolamine treated and control group was registered with decreased mRNA AChE, $\mathrm{BuChE}$, and BACE-1 levels, especially in the frontal cortex, suggesting the potential efficacy of this extract in this kind of pathologies [25, 28, 48-50].

This is only an example of the fact in the las decade some investigations have been carried out to demonstrate the potential pharmacological activity of Eryngium species extracts $[51,52]$.

\section{Cultivation}

Several genus Eryngium species have been widely used as edible plants in many countries and, consequently, mainly cultivated as an economic crop in tropical areas of the world [24, 28, 29]. In fact, numerous reports refer to the ideal and effective conditions for cultivating, harvesting as well as post-harvesting conditions of Eryngium species [24, 31, 53]. In the northern parts of Iran, E. caucasicum is one of the most important garden vegetables, which is mostly used in preparation of foodstuff, pickles, etc. $[28,29]$. It has been reported that some Eryngium species are easily cultivated in dry, sandy, well-drained soils, and full sun [24, 31, 53]. Root cuttings are a common protocol for propagation of Eryngium species [24, 31, 53-55], which can also be propagated using other plant cuttings [24, 31, 53-55]. In addition, there is a close correlation between Eryngium species growth and fertilizer levels in the soil [24, 31, 53-55]. However, Eryngium species are significantly endangered by plant diseases as well as insect attacks $[24,31,53-55]$.

\section{Phytochemistry}

Based on the review of the literature up to now, the aerial parts of Eryngium species have been reported to contain mainly saponins, flavonoids, and essential oil, while the underground parts contain triterpene saponins, monoterpene glycosides, phenolic compounds such as flavonoids and phenolic acids, coumarin derivatives, terpene aldehyde esters, acetylenes, essential oil, and oligosaccharides [56-60].

\section{Saponins}

The Eryngium species are the rich sources of triterpene saponins (Tables 1 and 2). Most of the saponins isolated from Eryngium species possess mainly hydroxylated oleanane-type aglycons such as A1-barrigenol (1), R1barrigenol (2), or barringtogenol C (3) (Fig. 2). In addition to these core structures, cameliagenin A (4), erynginol A (5) and B, betulinic acid, oleanolic acid, and steganogenin (6) having glucose, glucuronic acid, rhamnose, xylose, galactose, and arabinose moieties have been also found in various Eryngium species (Fig. 2). The general saponin structures possess generally acetic, angelic, dimethylacrylic, and tiglic acid substituents, located predominantly at $\mathrm{C} 21, \mathrm{C} 22$ or $\mathrm{C} 28$ positions. On the other hand, isovaleric, $n$-butyric, and methyl butyric acids occur relatively rare substituents. Saponin glycosides found in Eryngium species are usually monodesmosidic saponins, where the bidesmosidic ones contain sugar groups at C3 and C28 positions [59, 61-65]. 
Table 1 Triterpene saponins from Eryngium species isolated between 1970-1978

\begin{tabular}{|c|c|c|c|c|}
\hline Source & Sapogenol & Type of sapogenol & Plant part & Ref. \\
\hline \multirow[t]{4}{*}{ Eryngium planum L. } & Eryngiumgenin $\mathrm{A}$ & A1-barrigenol & Roots & [69] \\
\hline & Eryngiumgenin B & A1-barrigenol & & \\
\hline & Eryngiumgenin $C$ & R1- barrigenol & & \\
\hline & Eryngiumgenin D & R1- barrigenol & & \\
\hline Eryngium planum L. & Erynginol A & Barringtogenol C & Aerial & [70] \\
\hline Eryngium bromeliifolium Delar. & Oleanic acid type of sapogenols & Oleanic acid & Leaves & [66] \\
\hline \multirow[t]{6}{*}{ Eryngium planum L. } & Eryngiumgenin $\mathrm{F}$ & Barringtogenol C & Roots & [61] \\
\hline & Eryngiumgenin G & $\mathrm{n} / \mathrm{a}$ & & \\
\hline & Eryngiumgenin $\mathrm{H}$ & $\mathrm{n} / \mathrm{a}$ & & \\
\hline & Eryngiumgenin J & $\mathrm{n} / \mathrm{a}$ & & \\
\hline & Eryngiumgenin $\mathrm{K}$ & R1- barrigenol & & \\
\hline & Eryngiumgenin $\mathrm{L}$ & $\mathrm{n} / \mathrm{a}$ & & \\
\hline Eryngium bromeliifolium Delar. & Betulinic acid & & Leaves & [71] \\
\hline \multirow[t]{10}{*}{ Eryngium giganteum L. } & Giganteumgenin A & $\mathrm{n} / \mathrm{a}$ & Leaves & [67] \\
\hline & Giganteumgenin B & $\mathrm{n} / \mathrm{a}$ & & \\
\hline & Giganteumgenin C & Oleanic acid & & \\
\hline & Giganteumgenin D & $\mathrm{n} / \mathrm{a}$ & & \\
\hline & Giganteumgenin E & $\mathrm{n} / \mathrm{a}$ & & \\
\hline & Giganteumgenin G & $\mathrm{n} / \mathrm{a}$ & & \\
\hline & Giganteumgenin $\mathrm{H}$ & $\mathrm{n} / \mathrm{a}$ & & \\
\hline & Giganteumgenin K & $\mathrm{n} / \mathrm{a}$ & & \\
\hline & Giganteumgenin M & Barringtogenol C & & \\
\hline & Giganteumgenin N & R1- barrigenol & & \\
\hline Eryngium planum L. & Eryngiumgenin E & & Roots & [72] \\
\hline Eryngium bromeliifolium Delar. & Betulinic acid-3-O- $\beta$-glycoside & & Leaves & [73] \\
\hline Eryngium maritimum L. & $\begin{array}{l}\text { Eryngiumgenin C and sapogenols with A1-barrigenol, } \\
\text { R1-barrigenol and barringtogenol C structures }\end{array}$ & & Aerial parts & [74] \\
\hline \multirow[t]{4}{*}{ Eryngium amethystinum L. } & Main structure: Barringtogenol C & & & [68] \\
\hline & R1-barrigenol & & & \\
\hline & Erynginol A & & & \\
\hline & A1-barrigenol & & & \\
\hline Eryngium bromeliifolium Delar. & $\begin{array}{l}\text { 3-O-D-Glucopyranosyloleanolic acid-28-O-D-xsylopyranoside } \\
\text { (Saponin F) }\end{array}$ & & Leaves & [62] \\
\hline Eryngium planum L. & R1-barrigenol + an acid substituent and 2 glycopyranosyl moieties & & Roots & {$[75]^{a}$} \\
\hline
\end{tabular}

${ }^{a}$ This study was published in 1985 and it is the only study published between 1978-2002

The first phytochemical studies performed on the genus Eryngium starting from early 1970s were focused on their saponin content. According to the survey of the literature data published between 1970-1978 and in 1985 , the identification of the isolated compounds was limited to the absolute definition of the aglycons with possible positions of sugars and acyl substituents because of inadequate chemical facilities in structure elucidation techniques $[61,66-68]$. In this period, $E$. planum, E. amethystinum L., E. giganteum M. Bieb., and E. bromeliifolium F. Delaroche were studied in more detail. As summarized in Table 1, A1-barrigenol and R1-barrigenol-type of aglycons containing dimethylacrylic, angelic, and/or tyglic acids as the acid moieties were isolated from the roots of the E. planum, named as eryngiumgenine A-D [69] of which only aglycon types were identified and classified according to the $R_{f}$ values. Later, compound (5) was isolated from the aerial parts of this species [70]. Further phytochemical studies on the same plant were continued on the leaves and 
Table 2 Triterpene saponins from Eryngium species isolated after 2002

\begin{tabular}{|c|c|}
\hline Source & Saponins \\
\hline Eryngium foetidum (Linn) & 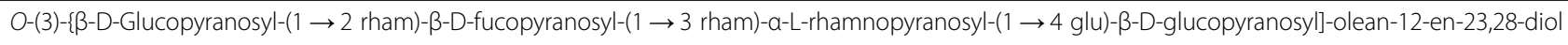 \\
\hline \multirow[t]{2}{*}{ Eryngium campestre L. } & 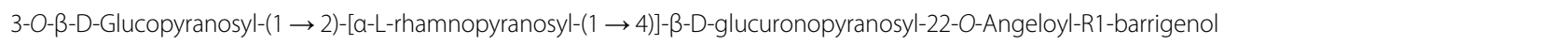 \\
\hline & 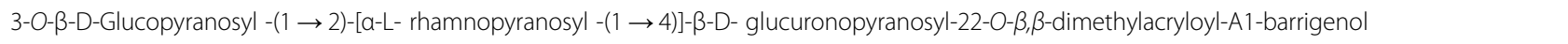 \\
\hline \multirow[t]{5}{*}{ Eryngium campestre L. } & 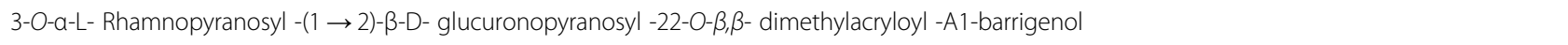 \\
\hline & 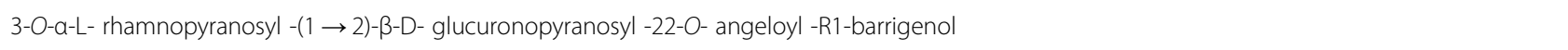 \\
\hline & 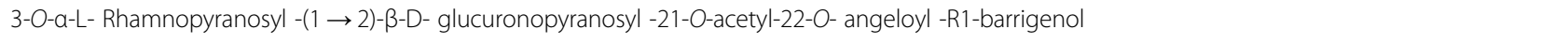 \\
\hline & 3-O-a-L- Rhamnopyranosyl -(1 $\rightarrow$ 2)- $\beta$-D- glucuronopyranosyl -21-O- acetyl -22-O- $\beta, \beta$ - dimethylacryloyl -R1-barrigenol \\
\hline & 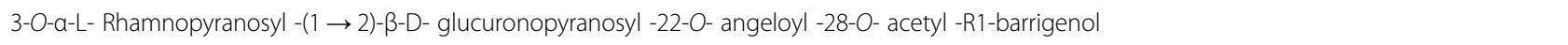 \\
\hline \multirow[t]{13}{*}{$\begin{array}{l}\text { Eryngium yuccifolium } \\
\text { Michx. }\end{array}$} & 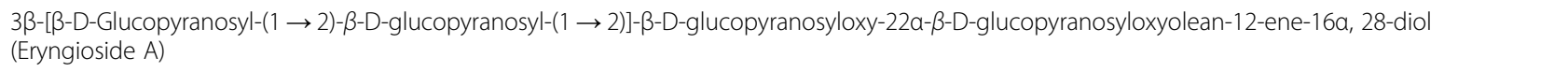 \\
\hline & 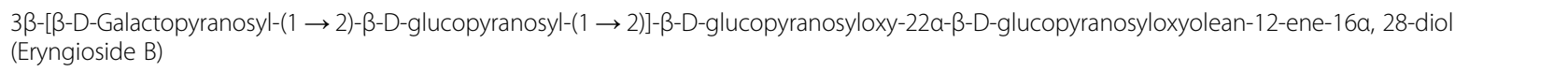 \\
\hline & 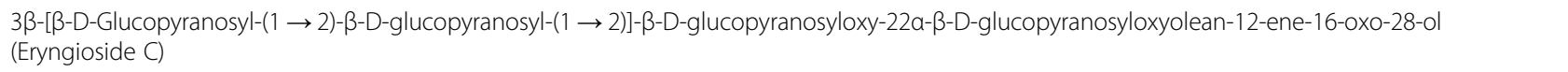 \\
\hline & 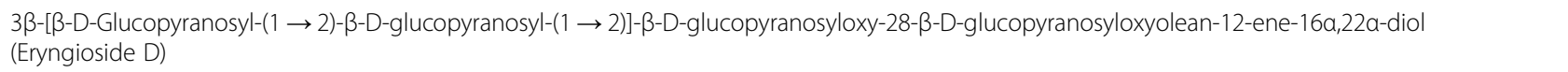 \\
\hline & 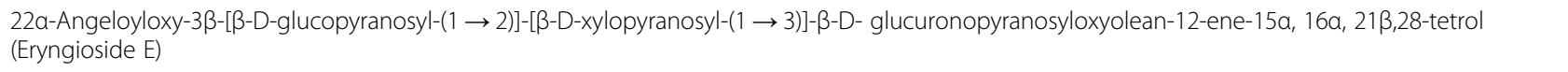 \\
\hline & 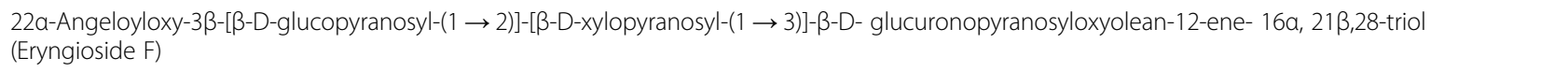 \\
\hline & 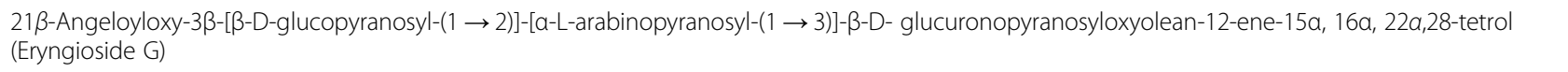 \\
\hline & 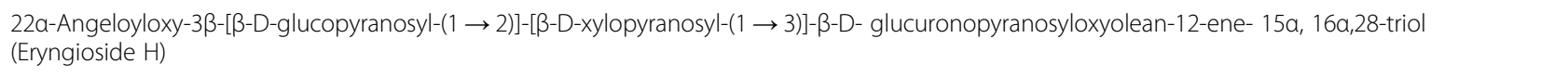 \\
\hline & 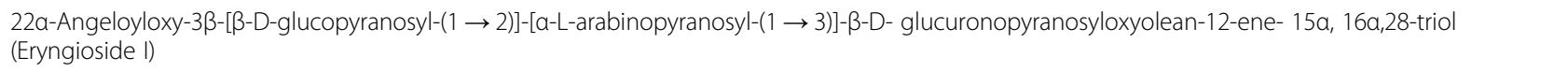 \\
\hline & 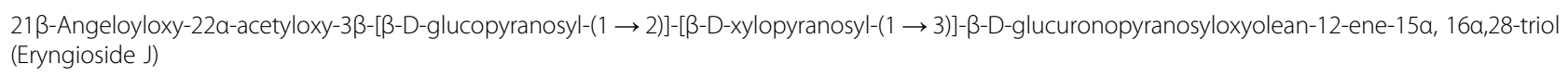 \\
\hline & 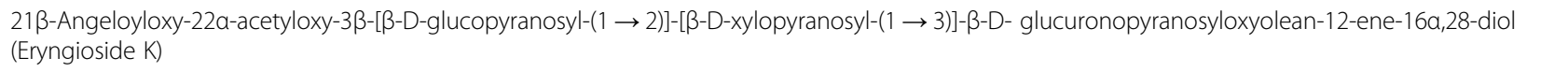 \\
\hline & 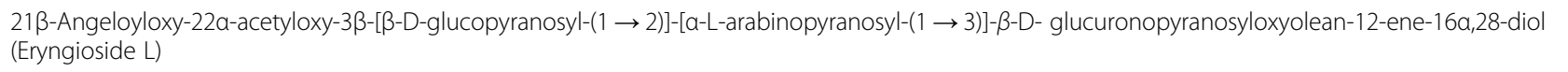 \\
\hline & Saniculasaponin III \\
\hline
\end{tabular}


Table 2 Triterpene saponins from Eryngium species isolated after 2002 (Continued)

Saniculasaponin II and II

Eryngium planum L. $\quad$ 3-O-B-D-Glucopyranosyl-( $\rightarrow$ - 2)-B-D-glucuronopyranosyl-21-O-acetyl-22-O-angeloyl-R1-barrigenol

3-O- $\beta$-D-Glucopyranosyl-(1 $\rightarrow$ 2)- $\beta$-D-glucuronopyranosyl-22-O-angeloyl-A1-barrigeno

Eryngium kotschyi Boiss.

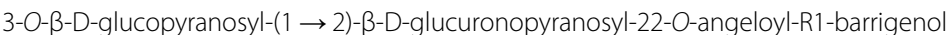

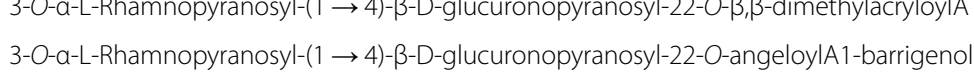

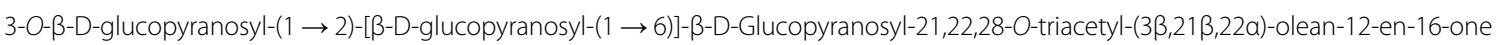

3-O-B-D-Glucopyranosyl-( $1 \rightarrow 2$ )- glucopyranosyl-22-O- $\beta$-D- glucopyranosylsteganogenin

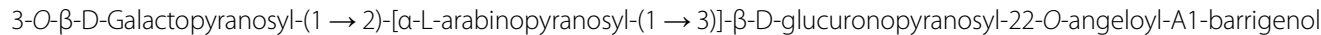

3-O-a-L-Rhamnopyranosyl-(1 $\rightarrow$ 4)-B-D-glucuronopyranosyloleanolic acid 


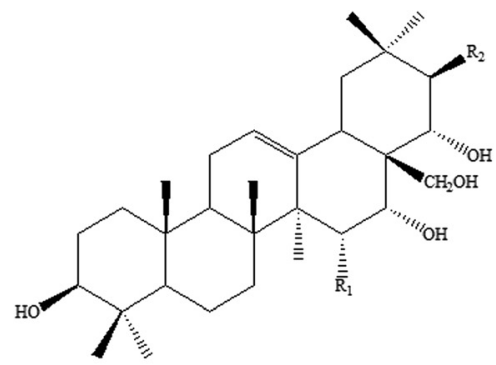

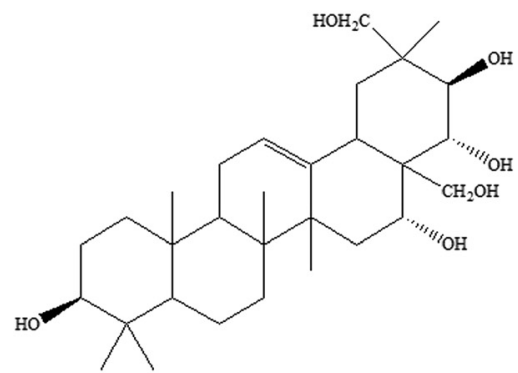

Erynginol A (5) $\begin{array}{lll}\text { Aglycon } & \mathrm{R}_{1} \quad \mathrm{R}_{2}\end{array}$

A1-barrigenol (1) $\quad \mathrm{OH} \quad \mathrm{H}$

R1-barrigenol (2) $\quad \mathrm{OH} \quad \mathrm{OH}$

Barringtogenol C (3) $\quad \mathrm{H} \quad \mathrm{OH}$

Camelliagenin A (4) $\quad \mathrm{H} \quad \mathrm{H}$<smiles>CC(C)(C)CC1C(O)=CC[C@]2(C)C1=CCC1C2CC[C@]2(C)C1CC[C@@H](O)C2(C)C</smiles>

Steganogenin (6)

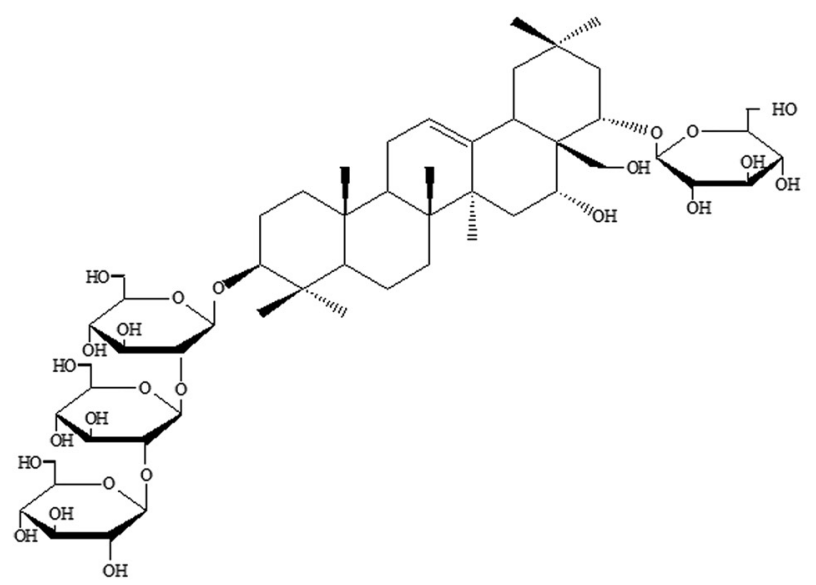

Eryngioside A (7)

Fig. 2 Saponin derivatives frequently found in Eryngium species

roots. These studies led to the isolation of a number of aglycons with (4), (3), (2) types possessing acetic, tyglic, butyric, and isovalerianic acids located at C16, C21, C22 or C28 positions [71, 72]. Isolation of saponin aglycones such as (2) and (3), and saponins bearing (2) and (3) skeletons, named as giganteumgenin A (7), B (8), C (9), D (10), E (11), G (12), H (13), K (14), M (15), and $\mathrm{N}$ (16) (Fig. 3), was achieved from the methanol extract of the leaves of E. giganteum [67]. Another phytochemical investigation on $E$. amethystinum led to isolation of the saponins with main structures in (1), (2), (3), and (5) [68]. Other sapogenols isolated from various Eryngium species until 1978 are listed in Table 1 [58, 62, 72-74]. Many years later, another R1-barringtogenol derivative with an acid moiety and two glycopyranosyl moieties was reported from E. planum roots by Voigt et al. in 1985 [75].

Most of the scientific articles concerning saponins of the genus Eryngium remained limited to the identification of the sapogenol type and determination of the acid or sugar substituents. After the late 70's, publications concerning the saponins from the genus Eryngium have become much more detailed as giving the whole structure elucidations as a result of the developments of the techniques used for structure elucidation. For 
<smiles>[R]C1C([R])C2([R])C(CC(C)(C)C([R])C2[R4])C2=CCC3C4(C)CCC(O)C(C)(C)C4CCC3(C)C23CC13</smiles>

\begin{tabular}{|c|c|c|c|c|c|c|}
\hline Sapogenol & & $\mathrm{R}_{1}$ & $\mathrm{R}_{2}$ & $\mathrm{R}_{3}$ & $\mathrm{R}_{4}$ & $\mathrm{R}_{5}$ \\
\hline $\begin{array}{l}\text { Giganteumgenin } \\
\text { (7) }\end{array}$ & $\mathbf{A}^{*}$ & $\begin{array}{l}\text {-O-Angeloyl } \\
\text {-O-Tigloyl } \\
\text {-O-Dimethylacryloyl }\end{array}$ & $-\mathrm{OCOCH}_{3}$ & $-\mathrm{CH}_{2} \mathrm{OCOCH}_{3}$ & $-\mathrm{OH}$ & $-\mathrm{H}$ \\
\hline $\begin{array}{l}\text { Giganteumgenin } \\
\text { (8) }\end{array}$ & $\mathbf{B}^{*}$ & $\begin{array}{l}\text {-O-Angeloyl } \\
\text {-O-Tigloyl } \\
\text {-O-Dimethylacryloyl }\end{array}$ & $-\mathrm{OCOCH}_{3}$ & $-\mathrm{CH}_{2} \mathrm{OH}$ & $-\mathrm{OH}$ & $-\mathrm{H}$ \\
\hline Giganteumgenin C (S & (9) & $-\mathrm{H}$ & $-\mathrm{H}$ & $-\mathrm{COOH}$ & $-\mathrm{H}$ & $-\mathrm{H}$ \\
\hline $\begin{array}{l}\text { Giganteumgenin } \\
\text { (10) }\end{array}$ & D & $-\mathrm{OCOC}_{4} \mathrm{H}_{7}$ & $-\mathrm{OCOCH}_{3}$ & $-\mathrm{CH}_{2} \mathrm{OH}$ & $-\mathrm{OH}$ & $-\mathrm{OCOCH}_{3}$ \\
\hline $\begin{array}{l}\text { Giganteumgenin } \\
\text { (11) }\end{array}$ & $\mathbf{E}^{*}$ & $\begin{array}{l}\text {-O-Angeloyl } \\
\text {-O-Tigloyl }\end{array}$ & $-\mathrm{OH}$ & $-\mathrm{CH}_{2} \mathrm{OH}$ & $-\mathrm{OH}$ & $-\mathrm{H}$ \\
\hline $\begin{array}{l}\text { Giganteumgenin } \\
\text { (12) }\end{array}$ & $\mathbf{G}^{*}$ & $\begin{array}{l}\text {-O-Angeloyl } \\
\text {-O-Tigloyl }\end{array}$ & $-\mathrm{OH}$ & $-\mathrm{CH}_{2} \mathrm{OH}$ & $-\mathrm{OH}$ & $-\mathrm{OH}$ \\
\hline $\begin{array}{l}\text { Giganteumgenin } \\
\text { (13) }\end{array}$ & $\mathbf{H}$ & $-\mathrm{OH}$ & $-\mathrm{OCOC}_{4} \mathrm{H}_{7}$ & $-\mathrm{CH}_{2} \mathrm{OH}$ & $-\mathrm{OH}$ & $-\mathrm{OH}$ \\
\hline $\begin{array}{l}\text { Giganteumgenin } \\
\text { (14) }\end{array}$ & $\mathbf{K}$ & $-\mathrm{OH}$ & $-\mathrm{OCOC}_{4} \mathrm{H}_{7}$ & $-\mathrm{CH}_{2} \mathrm{OH}$ & $-\mathrm{OH}$ & $-\mathrm{OH}$ \\
\hline $\begin{array}{l}\text { Giganteumgenin } \\
\text { (15) }\end{array}$ & M & $-\mathrm{OH}$ & $-\mathrm{OH}$ & $-\mathrm{CH}_{2} \mathrm{OH}$ & $-\mathrm{OH}$ & $-\mathrm{H}$ \\
\hline $\begin{array}{l}\text { Giganteumgenin } \\
\text { (16) } \\
\text { *Mixture compound }\end{array}$ & $\mathbf{N}$ & $-\mathrm{OH}$ & $-\mathrm{OH}$ & $-\mathrm{CH}_{2} \mathrm{OH}$ & $-\mathrm{OH}$ & $-\mathrm{OH}$ \\
\hline
\end{tabular}

Fig. 3 Structures of giganteumgenins A-N

instance, Anam [30] reported the full configuration of a new oleanane-type triterpene saponin structure (17) from the aerial parts of E. foetidum (Fig. 4). Kartal et al. [59] characterized two new triterpene saponins (18, 19) from the roots of E. campestre bearing $\mathbf{A}(\mathbf{1})$ and (2) types, and isolated five new triterpene saponins (20-24), one of which was (1) and the others with (2) main skeleton (Fig. 5) [63]. Another phytochemical study performed on the whole parts of E. yuccifolium was reported by Zhang et al. [64], who described the isolation and identification of 12 new polyhydroxylated triterpenoid saponins named as eryngiosides A-L (28-39) and two known triterpenoid saponins $\{21 \beta$-angeloyloxy- $3 \beta$ [ $\beta$-D-glucopyranosyl- $(1 \rightarrow 2)]$-[ $\beta$-D-xylopyranosyl- $(1 \rightarrow 3)]$ $\beta$-D-glucuronopyranosyloxyolean-12-ene-15 $\alpha, \quad 16 \alpha, 22 \alpha$, 28-tetrol (44) and saniculasaponin III (43)\} from the same species (Fig. 6). Furthermore, the root of E. yuccifolium afforded two new polyhydroxyoleanene saponins [eryngioside $M$ (40) and $N$ (41)], together with 15 known triterpenoid saponins $(28-39 ; 42-44)$ elucidated [47].

Further studies on the roots of E. planum led to the isolation of two R1- and one A1-barrigenol type of triterpene saponins [76], where 3-O- $\beta$-D- glucopyranosyl- $(1 \rightarrow 2)-\beta$ D-glucuronopyranosyl-21-O-acetyl-22-O-angeloyl-R1-barr igenol (25), 3-O- $\beta$-D-glucopyranosyl- $(1 \rightarrow 2)-\beta$-d-glucuronopyranosyl-22-O-angeloyl-A1-barrigenol (26) and 3-O- $\beta$ D-glucopyranosyl- $(1 \rightarrow 2)-\beta$-D-glucuronopyranosyl-22-O-a ngeloyl-R1-barrigenol (27) (Fig. 5) were also found to be present in the roots of E. planum [76]. Detailed phytochemical investigation on the roots of E. kotschyi Boiss., which is an endemic plant to Turkey, led to isolation of two known and four new triterpene saponins (45-48) (Fig. 7) [77]. 


\section{Flavonoids}

In an earlier study [78], isolation from E. planum of a new flavonol glycoside (identified as kaempferol-3-O-(6-O- $\beta-$ D-glucopyranosyl)- $\beta$-D-galactopyranoside) was reported. A phytochemical study on the aerial parts of $E$. campestre led to the characterization of a new acylated flavonol named as kaempferol 3-O- $\beta$-D-(2'-Z- $p$-coumaroylglucoside) (49) along with 10 known compounds including tiliroside (50), kaempferol $3-\mathrm{O}-\beta-\mathrm{D}$-glucosyde-7-O- $\alpha-\mathrm{L}-$ rhamnoside (51), rutin (52), kaempferol (53), quercetin (54), isorhamnetin (55), caffeic acid, chlorogenic acid, and mannitol [58] as well as luteolin 7-glucoside [79] of which some selected structures are given in Fig. 8. Using UHPLC-ESI-Q-TOF-MS technique, quantification of a number of flavonols (quercetin, kaempferol, isorhamnetin, and their derivatives) and naringenin rhamnoglucoside (a flavanone derivative) was performed in E. bourgatii Gouan by Cádiz-Gurrea et al. [48]. Hawas et al. [80] isolated 11 flavonoid glycosides (including isorhamnetin 3-O- $\alpha$ rhamnoside, isorhamnetin $3-\mathrm{O}-\beta$-galactoside, isorhamnetin 3 -O- $\beta$-glucoside, isorhamnetin $3-\mathrm{O}-\beta$-rutinoside, myricetin $3-\mathrm{O}-\beta$-galactoside 4'-methylether, myricetin 3 -O- $\beta$-glucoside 3 -methylether, myricetin $3-\mathrm{O}-\beta$-glucoside 4'-methylether, quercetin $3-\mathrm{O}-\beta$-glucoside, quercetin $3-\mathrm{O}-\beta$-glucuronide 4'-methylether, and rutoside) from the aerial parts of E. campestre. Then, most recently, Khalfallah et al. [36] characterized five flavonoid derivatives from the aerial parts of E. triquetrum Vahl. described as kaempferol 3-O- $\beta$-D-glucoside, kaempferol 3-O-[6" -O-E-p-coumaroyl]- $\beta$-D-glucopyranoside, kaempferol 3-O-[2", $6^{\prime \prime}-\mathrm{di}-O-E-p$-coumaroyl]$\beta$-D-glucoside, kaempferol 3-O-[ $\alpha$-L-rhamnosyl- $(6 \rightarrow$

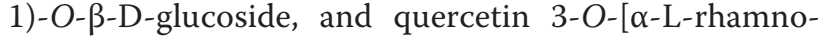
syl- $(6 \rightarrow 1)-O-\beta$-D-glucoside].

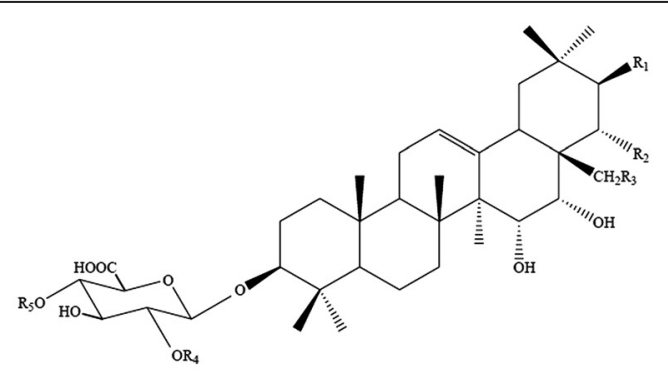

\begin{tabular}{|c|c|c|c|c|c|c|c|}
\hline $\begin{array}{l}\text { Plant } \\
\text { Name }\end{array}$ & Compound & $\mathbf{R}_{1}$ & $\mathbf{R}_{\mathbf{2}}$ & $\mathbf{R}_{3}$ & $\mathbf{R}_{4}$ & $\mathbf{R}_{5}$ & Ref. \\
\hline $\begin{array}{l}E . \\
\text { campestre }\end{array}$ & 18 & $\mathrm{OH}$ & Angeloyl & $\mathrm{OH}$ & Glycose & Rhamnose & 56 \\
\hline $\begin{array}{l}E . \\
\text { campestre }\end{array}$ & 19 & $\mathrm{H}$ & Dimethylacryloyl & $\mathrm{OH}$ & Glycose & Rhamnose & 56 \\
\hline $\begin{array}{l}E . \\
\text { campestre }\end{array}$ & 20 & $\mathrm{H}$ & Dimethylacryloyl & $\mathrm{OH}$ & Rhamnose & $\mathrm{H}$ & 60 \\
\hline $\begin{array}{l}E . \\
\text { campestre }\end{array}$ & 21 & $\mathrm{OH}$ & Angeloyl & $\mathrm{OH}$ & Rhamnose & $\mathrm{H}$ & 60 \\
\hline $\begin{array}{l}E . \\
\text { campestre }\end{array}$ & 22 & Acetyl & Angeloyl & $\mathrm{OH}$ & Rhamnose & $\mathrm{H}$ & 60 \\
\hline $\begin{array}{l}\text { E. } \\
\text { campestre }\end{array}$ & 23 & Acetyl & Dimethylacryloyl & $\mathrm{OH}$ & Rhamnose & $\mathrm{H}$ & 60 \\
\hline $\begin{array}{l}E . \\
\text { campestre }\end{array}$ & 24 & $\mathrm{OH}$ & Angeloyl & Acetyl & Rhamnose & $\mathrm{H}$ & 60 \\
\hline E. planum & 25 & Acetyl & Angeloyl & $\mathrm{OH}$ & Glycose & $\mathrm{H}$ & 73 \\
\hline E. planum & 26 & $\mathrm{H}$ & Angeloyl & $\mathrm{OH}$ & Glycose & $\mathrm{H}$ & 73 \\
\hline E. planum & 27 & $\mathrm{OH}$ & Angeloyl & $\mathrm{OH}$ & Glycose & $\mathrm{H}$ & 73 \\
\hline
\end{tabular}

Fig. 4 Structure of the (17) isolated from E. foetidum 


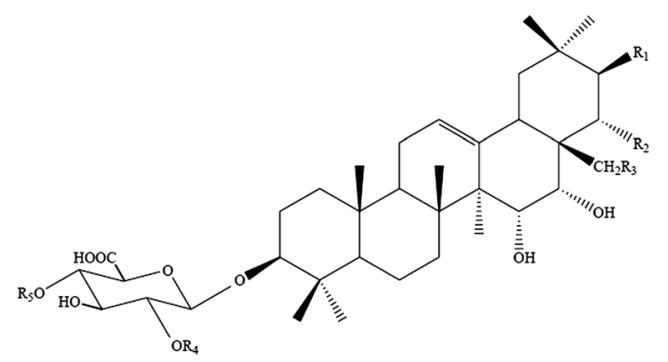

\begin{tabular}{|c|c|c|c|c|c|c|c|}
\hline $\begin{array}{l}\text { Plant } \\
\text { Name }\end{array}$ & Compound & $\mathbf{R}_{1}$ & $\mathbf{R}_{\mathbf{2}}$ & $\mathbf{R}_{\mathbf{3}}$ & $\mathbf{R}_{4}$ & $\mathbf{R}_{\mathbf{5}}$ & Ref. \\
\hline $\begin{array}{l}E . \\
\text { campestre }\end{array}$ & 18 & $\mathrm{OH}$ & Angeloyl & $\mathrm{OH}$ & Glycose & Rhamnose & 56 \\
\hline $\begin{array}{l}E . \\
\text { campestre }\end{array}$ & 19 & $\mathrm{H}$ & Dimethylacryloyl & $\mathrm{OH}$ & Glycose & Rhamnose & 56 \\
\hline $\begin{array}{l}E . \\
\text { campestre }\end{array}$ & 20 & $\mathrm{H}$ & Dimethylacryloyl & $\mathrm{OH}$ & Rhamnose & $\mathrm{H}$ & 60 \\
\hline $\begin{array}{l}E . \\
\text { campestre }\end{array}$ & 21 & $\mathrm{OH}$ & Angeloyl & $\mathrm{OH}$ & Rhamnose & $\mathrm{H}$ & 60 \\
\hline $\begin{array}{l}E . \\
\text { campestre }\end{array}$ & 22 & Acetyl & Angeloyl & $\mathrm{OH}$ & Rhamnose & $\mathrm{H}$ & 60 \\
\hline $\begin{array}{l}E . \\
\text { campestre }\end{array}$ & 23 & Acetyl & Dimethylacryloyl & $\mathrm{OH}$ & Rhamnose & $\mathrm{H}$ & 60 \\
\hline $\begin{array}{l}E . \\
\text { campestre }\end{array}$ & 24 & $\mathrm{OH}$ & Angeloyl & Acetyl & Rhamnose & $\mathrm{H}$ & 60 \\
\hline E. planum & 25 & Acetyl & Angeloyl & $\mathrm{OH}$ & Glycose & $\mathrm{H}$ & 73 \\
\hline E. planum & 26 & $\mathrm{H}$ & Angeloyl & $\mathrm{OH}$ & Glycose & $\mathrm{H}$ & 73 \\
\hline E. planum & 27 & $\mathrm{OH}$ & Angeloyl & $\mathrm{OH}$ & Glycose & $\mathrm{H}$ & 73 \\
\hline
\end{tabular}

Fig. 5 Saponins isolated from E. campestre and E. planum

\section{Coumarin derivatives}

The first coumarin derivatives isolated from E. campestre by Sticher \& Erdelmeier [56] were agasyllin (56), grandivittin (57), aegelinol benzoate (58), and aegelinol (59) which structures are given in Fig. 9 [56]. The isolation of marmesin tiglate [also known as nodakenetin (60), Fig. 10] together with metetoin, a nitrogenous compound, was achieved from E. ilicifolium Lam. [81].

\section{Phenolic acid derivatives}

Many Eryngium species have been reported to contain phenolic substances. Le Claire et al. [82] characterized chlorogenic (63), R-(+)-rosmarinic (61), and $R-(+)-3$ '- $O-\beta$ D-glucopyranosyl rosmarinic acids (62) in the roots of $E$. alpinum through medium pressure liquid chromatography
(MPLC) and preparative high pressure liquid chromatography (HPLC) (Fig. 11). Besides, the presence of R(+)-rosmarinic acid and $R-(+)-3$ '-O- $\beta$-D-glucopyranosyl rosmarinic acid was also shown in several Eryngium species which could be used as chemotaxanomic markers specific to this genus. Two new caffeic acid derivatives, i.e. 3,4-dihydroxyphenyl caffeate and (4- $\beta$ D-glucopyranosyloxy)-3-hydroxyphenyl caffeate, along with a new flavonoid (kaempferol-3-O-(2-O-trans- $p$ methoxycoumaroyl-6-O-trans- $p$-coumaroyl)- $\beta$-D-glucopyranoside) were isolated from the whole parts of $E$. yuccifolium in addition to following known compounds, i.e. caffeic acid and kaempferol-3-O-(2,6-di-O-trans-p-coumaroyl)- $\beta$-D-glucopyranoside [64]. Cádiz-Gurrea et al. [48] also revealed presence of cinnamic acid derivatives 


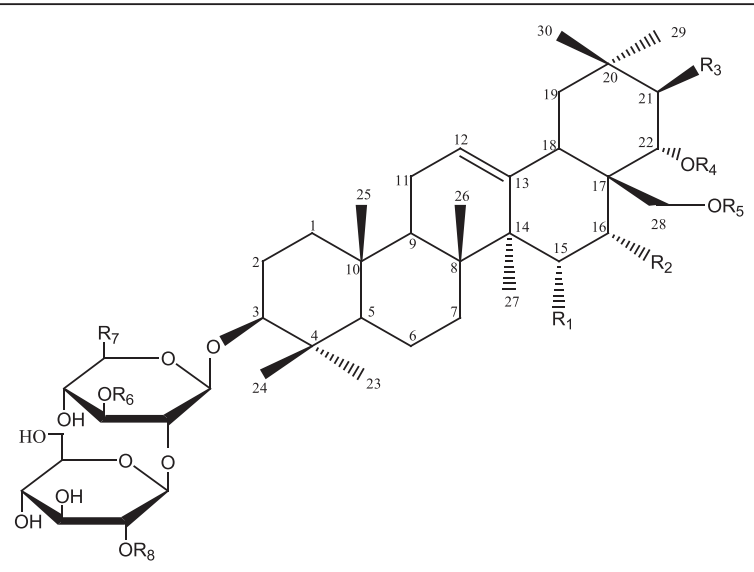

\begin{tabular}{lllllllll}
\hline & $\mathbf{R}_{\mathbf{1}}$ & $\mathbf{R}_{\mathbf{2}}$ & $\mathbf{R}_{\mathbf{3}}$ & $\mathbf{R}_{4}$ & $\mathbf{R}_{\mathbf{5}}$ & $\mathbf{R}_{\mathbf{6}}$ & $\mathbf{R}_{7}$ & $\mathbf{R}_{\mathbf{8}}$ \\
\hline Eryngioside A (28) & $\mathrm{H}$ & $\mathrm{OH}$ & $\mathrm{H}$ & Glycose & $\mathrm{H}$ & $\mathrm{H}$ & $\mathrm{CH}_{2} \mathrm{OH}$ & Glycose \\
Eryngioside B (29) & $\mathrm{H}$ & $\mathrm{OH}$ & $\mathrm{H}$ & Glycose & $\mathrm{H}$ & $\mathrm{H}$ & $\mathrm{CH}_{2} \mathrm{OH}$ & Galactose \\
Eryngioside C (30) & $\mathrm{H}$ & $\mathrm{O}$ & $\mathrm{H}$ & $\mathrm{Glycose}$ & $\mathrm{H}$ & $\mathrm{H}$ & $\mathrm{CH}_{2} \mathrm{OH}$ & $\mathrm{Glycose}$ \\
Eryngioside D (31) & $\mathrm{H}$ & $\mathrm{OH}$ & $\mathrm{H}$ & $\mathrm{H}$ & $\mathrm{Glycose}$ & $\mathrm{H}$ & $\mathrm{CH}_{2} \mathrm{OH}$ & $\mathrm{Glycose}$ \\
Eryngioside E (32) & $\mathrm{OH}$ & $\mathrm{OH}$ & $\mathrm{OH}$ & Angeloyl & $\mathrm{H}$ & Xylose & $\mathrm{COOH}$ & $\mathrm{H}$ \\
Eryngioside F (33) & $\mathrm{H}$ & $\mathrm{OH}$ & $\mathrm{OH}$ & Angeloyl & $\mathrm{H}$ & Xylose & $\mathrm{COOH}$ & $\mathrm{H}$ \\
Eryngioside G (34) & $\mathrm{OH}$ & $\mathrm{OH}$ & $\mathrm{O}-$ Angeloyl & $\mathrm{H}$ & $\mathrm{H}$ & Arabinose & $\mathrm{COOH}$ & $\mathrm{H}$ \\
Eryngioside H (35) & $\mathrm{OH}$ & $\mathrm{OH}$ & $\mathrm{H}$ & Angeloyl & $\mathrm{H}$ & Xylose & $\mathrm{COOH}$ & $\mathrm{H}$ \\
Eryngioside I (36) & $\mathrm{OH}$ & $\mathrm{OH}$ & $\mathrm{H}$ & Angeloyl & $\mathrm{H}$ & Arabinose & $\mathrm{COOH}$ & $\mathrm{H}$ \\
Eryngioside J (37) & $\mathrm{OH}$ & $\mathrm{OH}$ & $\mathrm{O}-$ Angeloyl & Acetyl & $\mathrm{H}$ & Xylose & $\mathrm{COOH}$ & $\mathrm{H}$ \\
Eryngioside K (38) & $\mathrm{H}$ & $\mathrm{OH}$ & $\mathrm{O}-$ Angeloyl & Acetyl & $\mathrm{H}$ & Xylose & $\mathrm{COOH}$ & $\mathrm{H}$ \\
Eryngioside L (39) & $\mathrm{H}$ & $\mathrm{OH}$ & $\mathrm{O}-$ Angeloyl & Acetyl & $\mathrm{H}$ & Arabinose & $\mathrm{COOH}$ & $\mathrm{H}$ \\
Eryngioside M (40) & $\mathrm{OH}$ & $\mathrm{OH}$ & $\mathrm{O}-$ Acetyl & Angeloyl & $\mathrm{H}$ & Xylose & $\mathrm{COOH}$ & $\mathrm{H}$ \\
Eryngioside N (41) & $\mathrm{OH}$ & $\mathrm{OH}$ & $\mathrm{OH}$ & Angeloyl & $\mathrm{H}$ & Arabinose & $\mathrm{COOH}$ & $\mathrm{H}$ \\
Saniculasaponin II (42) & $\mathrm{OH}$ & $\mathrm{OH}$ & $\mathrm{O}-$ Acetyl & Angeloyl & $\mathrm{H}$ & Arabinose & $\mathrm{COOH}$ & $\mathrm{H}$ \\
Saniculasaponin III (43) & $\mathrm{OH}$ & $\mathrm{OH}$ & $\mathrm{O}-$ Angeloyl & Acetyl & $\mathrm{H}$ & Arabinose & $\mathrm{COOH}$ & $\mathrm{H}$ \\
Compound 44 & $\mathrm{OH}$ & $\mathrm{OH}$ & $\mathrm{O}-$ Angeloyl & $\mathrm{H}$ & $\mathrm{H}$ & Xylose & $\mathrm{COOH}$ & $\mathrm{H}$ \\
\hline & & & & & & & & \\
\hline
\end{tabular}

Fig. 6 Saponins isolated from E. yuccifolium

(chlorogenic, rosmarinic, ferulic, caffeic acids, and their derivatives), benzoic acid derivatives ( $p$-hydroxybenzoic acid glucoside, arbutin, syringic acid, glucogallin, gentisic acid, and their derivatives) and various organic acids (gluconic, citric, quinic acids, and their derivatives) in E. bourgatii using hyphenated advance liquid chromatographic separation techniques. On the other hand, catechin, epicatechin, chlorogenic, gallic, and rosmarinic acids were determined quantitatively in the roots of E. palmatum Pančić \& Vis. [83]. In a similar study [60], chlorogenic, hydroxybenzoic, and caftaric acids were detected in $E$. bornmuelleri Nábělek along with trace amounts of caffeic, ferulic, and rosmarinic acids.

\section{Other types of compounds}

Drake and Lam [84] reported the isolation of falcarinone, a widespread acetylenic compound occurring in Apiaceae. 6Pentyl-2-[2-oxo-butin(3)-yliden]-tetrahydropyrane from the aerial parts and roots of $E$. bourgatti and the acetylenic compounds identified as $Z$ and $E$ isomers of the same compound were also obtained from this species in addition to falcarinone, falcarinolone, falcarinol, and scopoletin [85]. Further phytochemical studies ensued in the characterization of two new monoterpene glycosides of the cyclohexenone type, elucidated as 3-( $\beta$-D-glucopyranosyloxymethyl)-2,4,4-trimethyl-2,5-cyclohexadien-1-one and 3-( $\beta$-D-glucopyranosyloxymethyl)-2,4,4-trimethyl-2- 

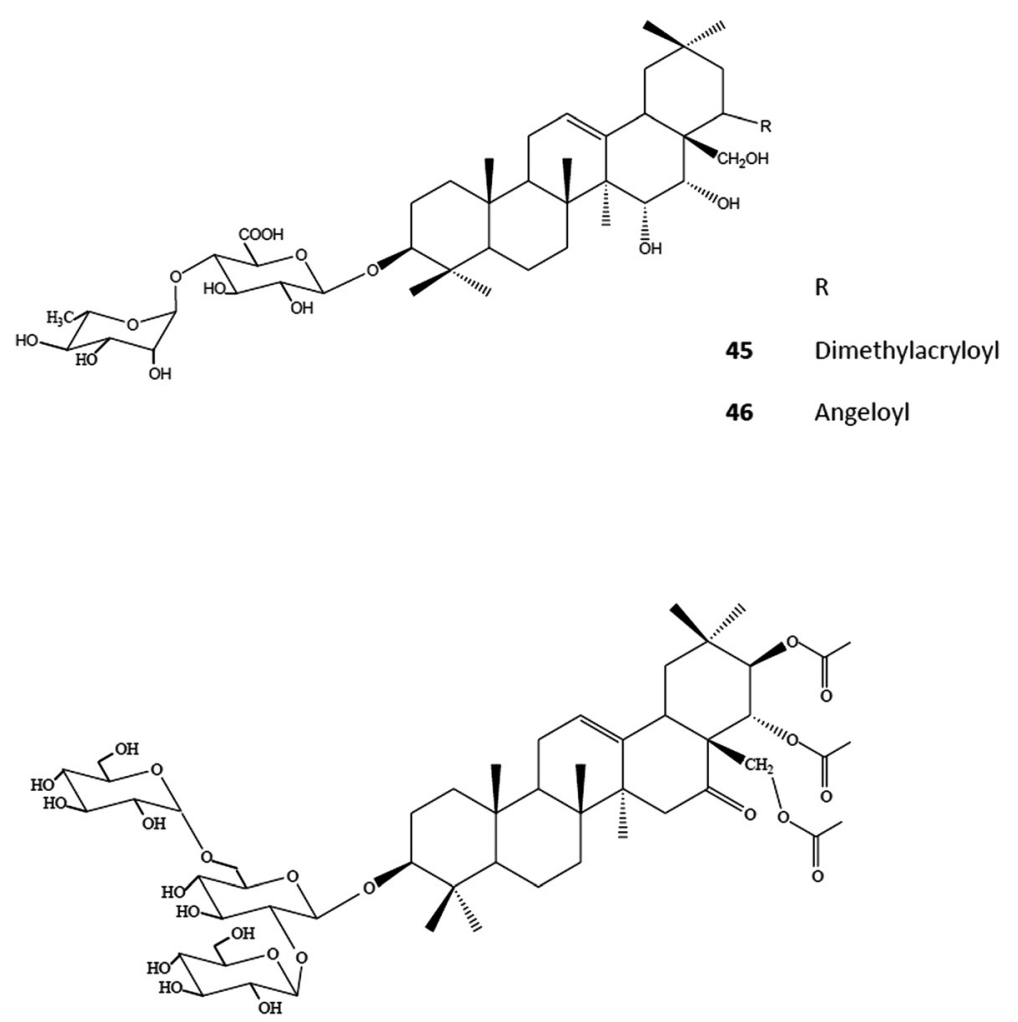

47

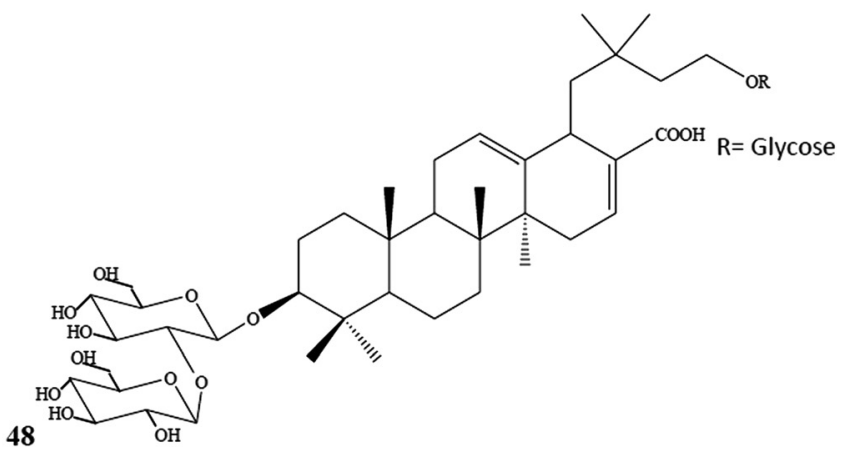

Fig. $\mathbf{7}$ Chemical structure of triterpene saponins

cyclohexen-1-one [57]. An unusual sesquiterpene whose structure was established as 1-n-propyl-perhydronaphthaline 1,2,4a,5,6,7,8,8a-octahydro-4-methyl-1-propyl-naphthalene-7-carbaldehyde was reported from the aerial parts of E. creticum [86], while new ester derivatives, characterized as cis-chrysanthenyl hexanoate and cis-chrysanthenyl octanoate, were isolated from E. planum [87].

In order to find out the compounds possibly responsible for the anti-inflammatory activity of E. foetidum, composition of the hexane extract from the leaves of this species was subjected to GC and GC-MS analyses [88]. Hereby, $\alpha$-cholesterol, brassicasterol, campesterol, stigmasterol (as the main component, $95 \%$ ), clerosterol, $\beta$-sitosterol, $\Delta_{5}$-avenasterol, $\Delta_{5} 24$-stigmastadienol, and $\Delta_{7}$-avenasterol were detected in the extract. Muckensturm et al. [89] reported a phytochemical study performed on the diethyl ether extracts from E. giganteum, E. variifolium Coss., E. planum, and E. maritimum. Chromatographic separation techniques applied to the seed extract of E. giganteum led to the elucidation of a new nor-sesquiterpene hydrocarbon, i.e. 15-nor- $\alpha$-muurolene (gigantene), and germacrene-D, trans- $\beta$-farnesene, 15 -oxy- $\alpha$-muurolene, 15 -hydroxy- $\alpha$ muurolene, ledol, and spatulenol. Similar studies on the seeds of the E. planum gave way to isolation of cis-chrysanthenyl acetate, while isoferulyl senecioate and 2,3,4-trimethylbenzaldehyde were obtained from the leaves of $E$. variifolium. Nevertheless, it should be noted that the latter compound was considered as artifact by these authors. In 


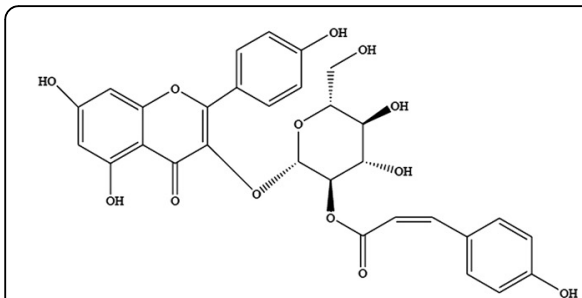

Kaempferol 3-O-ß-D-(2'-Z-p-coumaroylglucoside) (49)

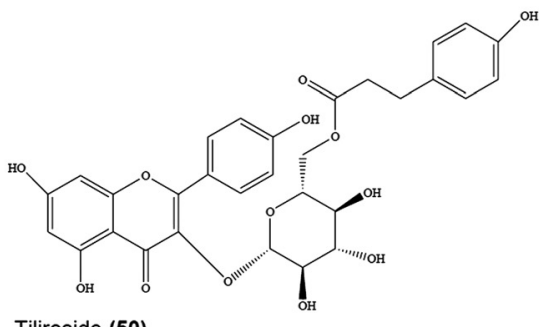

Tiliroside (50)<smiles>C[C@@H]1O[C@H](Cc2c(-c3ccc(O)cc3)oc3cc(O[C@@H]4O[C@H](CO)[C@@H](C)[C@H](O)[C@H]4O)cc(O)c3c2=O)[C@H](O)[C@@H](O)[C@@H]1O</smiles>

Kaempferol 3-O- $\beta$-D-glucosyde-7-O- $\alpha$-L-rhamnoside (51)

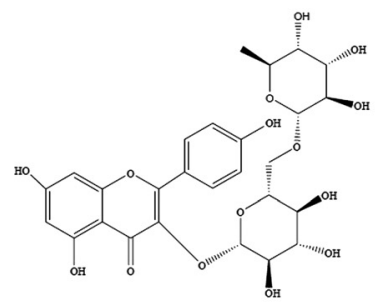

Rutin (52)<smiles>Cc1ccc(-c2oc3cc(O)cc(Cl)c3c(=O)c2O)cc1</smiles>

Kaempherol (53)<smiles>O=c1c(O)c(-c2ccc(O)c(O)c2)oc2cc(Br)cc(Cl)c12</smiles>

Quercetin (54)

Fig. 8 Selected flavonoids (49-55) isolated from E. campestre

another study [90], E. foetidum was subjected to isolation procedures which finally afforded trans-2-dodecanal (eryngial) in pure form.

\section{Essential oil}

Essential oil compositions of numerous Eryngium species have been investigated by many researchers. As illustrated

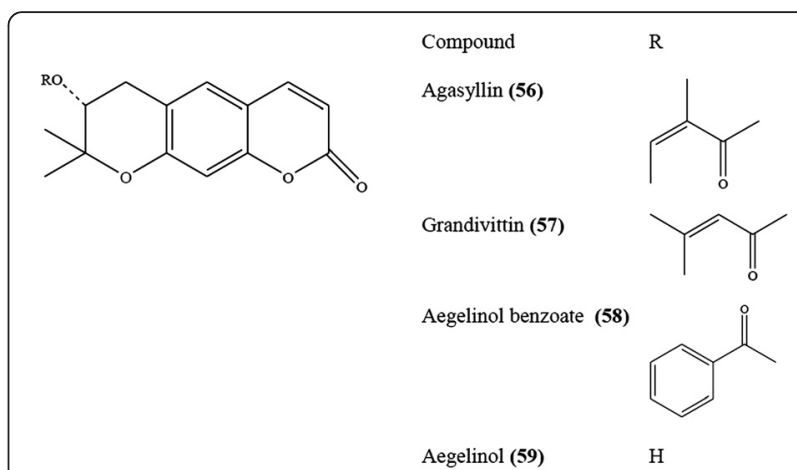

Fig. 9 Some coumarin derivatives isolated from E. campestre

with their corresponding references in Table 3, a great interspecies variation could be easily observed. However, the most common monoterpenes analyzed in different plant parts of Eryngium species have been as follows; germacrene D, $\alpha$-pinene, caryophyllene, muurolene, $\alpha$ - and $\beta$ selinene, limonene, $\alpha$ - and $\beta$-bisabolol, etc., whereas many hydrocarbons and some aromatics such as trimethylbenzaldehyde and dodecenal have been detected.

\section{Antimicrobial effects of the genus Eryngium plants}

Plants are known to produce antimicrobial substances [10], which act as plant defense mechanisms and protect them against abiotic and biotic stresses. These antimicrobial agents, which are often characterized by low adverse effects and wide spectrum activities, belong to many chemical classes such as phenolics and polyphenolics, terpenoids, alkaloids, lectins, polypeptides, and polyacetylenes [11].

Among the plants belonging to the genus Eryngium, some species exhibit considerable antimicrobial activity against gram-positive and gram-negative bacteria, some species of fungi and yeasts and viruses. Within this genus, the most studied species has been E. foetidum, which is cultivated across South Asia and Europe, Tropical Africa, and Pacific islands. As regards the antibacterial activity of E. foetidum extracts, the literature data are conflicting. In fact, in 2003, Alzoreky and Nakahara reported that the acetone and buffered methanol extracts obtained from $E$. foetidum leaves did not show any antibacterial activity against Escherichia coli, Salmonella

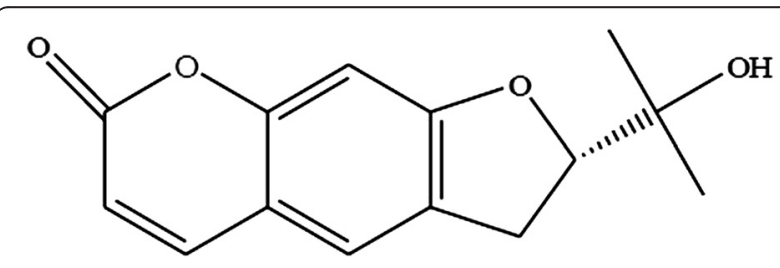

Fig. 10 Nodakenetin (60) isolated from E. ilicifolium 


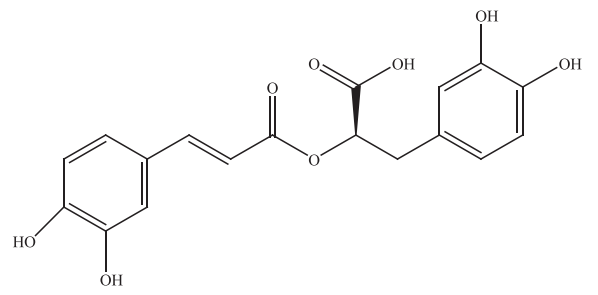

Compound

Rosmarinic acid (61)

$R$-(+)-3'-O- $\beta$-D-glucopyranosyl rosmarinic acid (62) $\beta$-D-glucopyranosyl

Fig. 11 Some phenolic acid derivatives found in E. alpinum

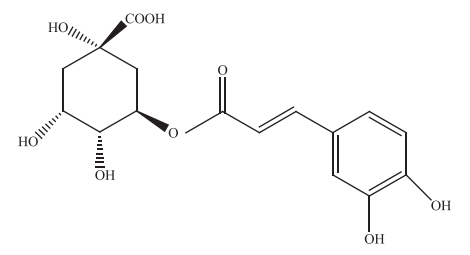

Chlorogenic acid (63) infantis, Listeria monocytogenes Tottori, Staphylococcus aureus and Bacillus cereus [91].

More recently, Ndip et al. showed that the methanolic extract form E. foetidum leaves showed moderate antibacterial activity against 6 clinical strains of Helicobacter pylori out of 15 tested strains, using the disk diffusion technique as antibacterial susceptibility test [92]. Besides antibacterial activity, E. foetidum was tested for its antiplasmodial activity, using chloroquine as positive control to evaluate the sensitivity of susceptible Plasmodium falciparum strains. The leaf extracts were prepared using hexane, dichloromethane, and methanol to obtain three extracts. The dried extracts were then dissolved in DMSO to give a stock solution at $10 \mathrm{mg} / \mathrm{mL}$, used for the biological tests. The results indicated that E. foetidum showed low in vitro antiplasmodial activity against P. falciparum, with an IC50 value of $25 \mu \mathrm{g} / \mathrm{mL}$ [93].

Another species belonging to genus Eryngium, is E. maritimum, which is a wild perennial species growing on sand beaches in West Europe, the Mediterranean basin, and the Black Sea and has been used for its diuretic, stimulant, cystotonic, stone inhibitor, aphrodisiac, expectorant, and anthelmintic properties (http://www.botanicals.com, http://www.crescentbloom.com). The essential oil, obtained from hydrodistillation of the aerial parts, was found to contain a known sesquiterpene (muurol-9-en-15-al) and three new oxygenated sesquiterpenes with a muuro-

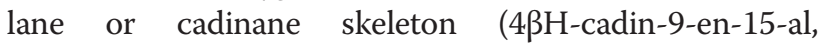
$4 \beta \mathrm{Hmuurol}-9$-en-15-ol, and $4 \beta \mathrm{H}$-cadin-9-en-15-ol), The sesquiterpenoid-rich fraction was tested in vitro using the agar diffusion method and the minimum inhibitory concentration (MIC) in the liquid phase against L. monocytogenes and E.coli. The finding revealed that the antibacterial activity of the new oxygenated sesquiterpenes against the tested bacteria, with an inhibition diameter higher than $15 \mathrm{~mm}$ and a MIC value lower than $90 \mu \mathrm{g} / \mathrm{mL}$ [94].

Another investigation on E. maritimun showed that the leaf hydromethanolic extract fractionated into a polar (aqueous) and apolar (chloroformic) fraction and tested using the microdilution method against food- borne pathogens and clinical isolates, exhibited antimicrobial activity. The tested Gram-positive bacteria were S. aureus subsp. aureus, Micrococcus luteus, $L$. monocytogenes and B. cereus. The Gram-negative bacteria were two strains of Salmonella (S. enterica subsp. arizonae and $S$. enterica subsp. montevideo), three strains of Pseudomonas ( $P$. aeruginosa, P. fluorescens, and P. marginalis), E. coli, and Erwinia carotovora subsp. carotovora, and a yeast (Candida albicans). According to the data obtained, the fractions resulted to be active against all bacteria with the exception of L. monocytogenes, while the most sensitive bacteria were $P$. aeruginosa and P. fluorescens, with MIC values of 1 and $2 \mu \mathrm{g} / \mathrm{mL}$ for the polar and apolar fractions, respectively [95].

The essential-oil composition and antimicrobial activity of three other species belonging to genus Eryngium (E. creticum, E. campestre, and E. thorifolium), whose infusions obtained from the aerial and root parts are commonly used in Turkish folk medicine as antitussive, diuretic, stimulant, and aphrodisiac, were studied [96]. The composition of E. thorifolium was found to be rich in $\alpha$-pinene, a known antibacterial terpenic compound, which was present in fewer amounts in the other two species. Differently, E. creticum was found to be rich in hexanal, which was present in less amount in E. thorifolium and was not detected in E. campestre. Antibacterial activity of the essential oils was tested with the disc diffusion method against nine clinical strains of methicillin-resistant S. aureus (MRSA). The essential oil obtained from $E$. thorifolium, which caused an inhibition zone ranging from 13 to $19 \mathrm{~mm}$ (similar to that exhibited by vancomycin and oregano essential oil tested at $10 \mu \mathrm{L} /$ disc and $5 \mu \mathrm{L} /$ disc, respectively), was demonstrated to be the most active species [37]. As aforementioned, many Eryngium species, e.g. E. maritimum exerted antifungal activity against $C$. albicans and other strains. Especially, the essential oil obtained by water distillation of the aerial parts of E. duriaei Gay ex Boiss subsp. juresianum (M.Laínz) M. Laínz was tested for its antifungal activity against 13 fungi, among which there were seven dermatophyte species (Microsporum canis FF1, 
Table 3 The major components in the essential oils of various Eryngium species

\begin{tabular}{|c|c|c|c|}
\hline Plant Name & Plant part & Major components & Ref. \\
\hline \multirow[t]{3}{*}{ Eryngium alpinum L. } & \multirow[t]{3}{*}{ Aerial parts } & Caryophyllene oxide (21.6 \%) & \multirow[t]{3}{*}{ [98] } \\
\hline & & Bicyclogermacrene (11.8\%) & \\
\hline & & Germacrene D (10.3 \%) & \\
\hline \multirow[t]{12}{*}{ Eryngium amethystinum L. } & \multirow[t]{3}{*}{ Aerial parts } & $\beta$-Caryophyllene (19.7\%) & \multirow[t]{3}{*}{ [98] } \\
\hline & & a-Bisabolol (7.9 \%) & \\
\hline & & 2,3,6-Trimethylbenzaldehyde (7.9\%) & \\
\hline & \multirow[t]{3}{*}{ Leafy parts of the shoots } & a-Pinene (11.8 \%) & \multirow[t]{9}{*}{ [99] } \\
\hline & & 2,3,6-Trimethylbenzaldehyde (24.7 \%) & \\
\hline & & Germacrene D (31.3 \%) & \\
\hline & \multirow[t]{3}{*}{ Inflorescence } & a-Pinene (25.6 \%) & \\
\hline & & 2,3,6- Trimethylbenzaldehyde (22.0 \%) & \\
\hline & & Germacrene D (14.5 \%) & \\
\hline & \multirow[t]{3}{*}{ Fruit } & a-Pinene (17.0 \%) & \\
\hline & & 2,3,6-Trimethylbenzaldehyde (16.9 \%) & \\
\hline & & Germacrene D (7.6 \%) & \\
\hline \multirow[t]{4}{*}{ Eryngium billardieri F. Delaroche } & \multirow[t]{4}{*}{ Aerial parts } & a-Muurolene (42.0\%) & \multirow[t]{4}{*}{ [39] } \\
\hline & & $\beta$-Gurjunene (17.0\%) & \\
\hline & & $\delta$-Cadinene (6.2\%) & \\
\hline & & Valencene (5.7 \%) & \\
\hline \multirow[t]{7}{*}{ Eryngium bourgatii Gouan } & \multirow[t]{2}{*}{ Inflorescence } & Phyllocladene (37.6 \%) & \multirow[t]{7}{*}{ [13] } \\
\hline & & Bicyclogermacrene (15.1\%) & \\
\hline & \multirow[t]{3}{*}{ Stems \& leaves } & Phyllocladene (20.4 \%) & \\
\hline & & $\gamma$-Muurolene (11.8\%) & \\
\hline & & (E)-Caryophyllene (10.1%) & \\
\hline & \multirow[t]{2}{*}{ Roots } & $\gamma$-Muurolene (15.4\%) & \\
\hline & & Phyllocladene (15.0 \%) & \\
\hline \multirow[t]{9}{*}{ Eryngium bungei Boiss. } & \multirow[t]{4}{*}{ Aerial parts } & Cumin alcohol (55.3 \%) & \multirow[t]{4}{*}{ [100] } \\
\hline & & Terpinolene (14.6\%) & \\
\hline & & Carvacrol (8.9\%) & \\
\hline & & Limonene (7.5 \%) & \\
\hline & \multirow[t]{5}{*}{ Aerial parts } & Borneol (44.4 \%) & \multirow[t]{5}{*}[101]{} \\
\hline & & Isobornyl formate (14.7\%) & \\
\hline & & Isoborneol (9.2 \%) & \\
\hline & & 1,8-Cineol (9.1 \%) & \\
\hline & & Camphor (7.9\%) & \\
\hline \multirow[t]{6}{*}{ Eryngium caeruleum M.B. } & \multirow[t]{2}{*}{ Aerial parts } & Limonene (60.5 \%) & \multirow[t]{2}{*}[102]{} \\
\hline & & $\delta$-3-Carene (13.0\%) & \\
\hline & Aerial parts & Cyclobuta[1-4]dicycloocten Hexadecahydro (47.03 \%) & [35] \\
\hline & & $n$-Hexadecanoic acid (11.16\%) & \\
\hline & & Limonene (4.23 \%) & \\
\hline & & Cis-a-bisabolene (2.14 \%) & \\
\hline
\end{tabular}


Table 3 The major components in the essential oils of various Eryngium species (Continued)

\begin{tabular}{|c|c|c|c|}
\hline \multirow[t]{6}{*}{ Eryngium campestre L. } & \multirow[t]{4}{*}{ Inflorescence } & Germacrene D (30.3-40.3 \%) & \multirow[t]{6}{*}{ [103] } \\
\hline & & $\beta$-Curcumene (0.7-22.2\%) & \\
\hline & & Myrcene (3.0-21.7 \%) & \\
\hline & & (E)- $\beta$-Farnesene (0.1-19.0\%) & \\
\hline & \multirow[t]{2}{*}{ Stems \& leaves } & Germacrene D (31.1-42.4 \%) & \\
\hline & & Myrcene (0.5-23.15 \%) & \\
\hline \multirow[t]{6}{*}{ Eryngium caucasicum Trautv. } & \multirow[t]{3}{*}{ Leaves (coastal samples) } & 4(5)-Acetyl-1H-imidazole (63.6 \%) & \multirow[t]{6}{*}{ [104] } \\
\hline & & Thymol (13.9\%) & \\
\hline & & $\beta$-Sesquiphellandrene (10.0\%) & \\
\hline & \multirow[t]{3}{*}{ Leaves (hill slope samples) } & $\beta$-Sesquiphellandrene (44.3\%) & \\
\hline & & Limonene (20.1\%) & \\
\hline & & Trans- $\beta$-Farnesene (14.1 \%) & \\
\hline \multirow[t]{9}{*}{ Eryngium corniculatum Lam. } & \multirow[t]{4}{*}{ Inflorescence } & 2,4,6-Trimethylbenzaldehyde (50.8\%) & \multirow[t]{9}{*}{ [105] } \\
\hline & & a-Pinene (4.0\%) & \\
\hline & & Crystanethylacetate (4.0\%) & \\
\hline & & 2,4,5-Trimethylbenzaldehyde (\%3.3) & \\
\hline & \multirow[t]{2}{*}{ Stems \& leaves } & 2,4,6-Trimethylbenzaldehyde (50.0 \%) & \\
\hline & & 2,4,5-Trimethylbenzaldehyde (3.8 \%) & \\
\hline & \multirow[t]{3}{*}{ Roots } & 2,4,6-Trimethylbenzaldehyde (29.8\%) & \\
\hline & & Phyllocladene isomer (13.0\%) & \\
\hline & & (E)-Nerolidol (9.4 \%) & \\
\hline \multirow[t]{3}{*}{ Eryngium creticum Lam. } & \multirow[t]{3}{*}{ Flowering aerial parts } & Hexanal (52.9 \%) & \multirow[t]{3}{*}[37]{} \\
\hline & & Heptanal (13.9\%) & \\
\hline & & Octane (8.95\%) & \\
\hline \multirow[t]{5}{*}{ Eryngium duriaei subsp. juresianum (M. Laínz) M. Laínz } & \multirow[t]{5}{*}{ Aerial parts } & a-Neocallitropsene (26.0\%) & \multirow[t]{5}{*}{ [106] } \\
\hline & & Isocaryophyllen-14-al (16.2 \%) & \\
\hline & & 14-Hidroxy- $\beta$-caryophyllene (13.4 \%) & \\
\hline & & Caryophyllene oxide (7.6\%) & \\
\hline & & E- $\beta$-Caryophyllene (6.3\%) & \\
\hline \multirow[t]{3}{*}{ Eryngium expansum $\mathrm{F}$. Muell. } & \multirow[t]{3}{*}{ Aerial parts } & 7-Epi-Selinene (38.3\%) & \multirow[t]{3}{*}{ [107] } \\
\hline & & Cis- $\beta$-Guaiene (10.8\%) & \\
\hline & & 2,3,6-Trimethylbenzaldehyde (8.0 \%) & \\
\hline \multirow[t]{13}{*}{ Eryngium foetidum L. } & \multirow[t]{3}{*}{ Aerial parts } & 2,3,6-Trimethylbenzaldehyde (5.5-23.7\%) & \multirow[t]{3}{*}{ [108] } \\
\hline & & (E)-2-Dodecenal (15.9-37.5 \%) & \\
\hline & & (E)-2-Tetradecenal (18.7-25.3\%) & \\
\hline & Aerial parts & 2,4,5-Trimethylbenzaldehyde (27.7\%) & [109] \\
\hline & & (E)-2-Dodecenal (27.5 \%) & \\
\hline & & Carotol (8.8 \%) & \\
\hline & & 3-Dodecenal (5.2 \%) & \\
\hline & Aerial parts & (E)-2-Dodecenal (57.79-67.08 \%) & [110] \\
\hline & & Lauraldehyde (7.04-11.53 \%) & \\
\hline & & 13-Tetradecenal (8.99-9.03\%) & \\
\hline & Leaves & 2,4,5-Trimethylbenzaldehyde (20.53\%) & [111] \\
\hline & & Hexadecanoic acid (12.05 \%) & \\
\hline & & Carotol (9.94 \%) & \\
\hline
\end{tabular}


Table 3 The major components in the essential oils of various Eryngium species (Continued)

\begin{tabular}{|c|c|c|c|}
\hline & Roots & Duraldehyde (37.60-53.14 \%) & [110] \\
\hline & & 13-Tetradecenal (7.22-13.16\%) & \\
\hline & & (E)-2-Dodecenal (7.14-11.62 \%) & \\
\hline & & Falcarinol (3.44-8.06 \%) & \\
\hline \multirow[t]{6}{*}{ Eryngium glaciale Boiss. } & Inflorescence & Phyllocladene isomer (43.5 \%) & [112] \\
\hline & & (E)-Caryophyllene (15.2 \%) & \\
\hline & & Valencene (11.5 \%) & \\
\hline & Stems \& leaves & Phyllocladene isomer (41.3\%) & \\
\hline & Roots & Phyllocladene isomer (49.4\%) & \\
\hline & & Linalool (19.1 \%) & \\
\hline \multirow[t]{10}{*}{ Eryngium maritimum L. } & Aerial parts & Spathulenol (18.99 \%) & [77] \\
\hline & & Caryophyllene oxide (8.18\%) & \\
\hline & Aerial parts & Germacrene D (10.4 \%) & [113] \\
\hline & & 2,4,5-Trimethylbenzaldehyde (8.3 \%) & \\
\hline & Roots & Germacrene D (15.9\%) & \\
\hline & & 2,4,5-Trimethylbenzaldehyde (6.7 \%) & \\
\hline & Aerial parts & Germacrene D (13.7-45.9 \%) & [114] \\
\hline & & 4ßH-Cadin-9-en-15-al (18.4-27.6 \%) & \\
\hline & & 4ßH-Cadin-9-en-15-ol (2.2-14.3 \%) & \\
\hline & & 4ßH-Muurol-9-en-15-al (4.3-9.3 \%) & \\
\hline \multirow[t]{5}{*}{ Eryngium pandanifolium Cham. et Schlecht } & Leaves & Bornyl acetate (20.8\%) & [107] \\
\hline & & $\beta$-Selinene (13.8\%) & \\
\hline & & a-Selinene (11.3\%) & \\
\hline & Fruit & Octanal (11.5\%) & \\
\hline & & $\beta$-Selinene (9.2 \%) & \\
\hline \multirow[t]{3}{*}{ Eryngium palmatum } & Roots & Octanal (31.7 \%) & [115] \\
\hline & & Curcumene (5.9\%) & \\
\hline & & 2,3,6-Trimethylbenzaldehyde (5.4\%) & \\
\hline \multirow[t]{2}{*}{ Eryngium paniculatum Cav. } & Inflorescence & (E)-Anethole (52.6 \%) & [116] \\
\hline & & a-Pinene (19.1\%) & \\
\hline \multirow[t]{7}{*}{ Eryngium planum L. } & Inflorescence & Cis-Chrysanthenyl acetate (43.2 \%) & [117] \\
\hline & Stalk leaves & Limonene (14.7 \%) & \\
\hline & & $\beta$-Pinene (9.8 \%) & \\
\hline & Rosette leaves & Bornyl acetate (18.1 \%) & \\
\hline & & Limonene (11.3 \%) & \\
\hline & & Terpinen-4-ol (10.9 \%) & \\
\hline & Roots & Falcarinol (64.4 \%) & \\
\hline \multirow[t]{5}{*}{ Eryngium rostratum Cav. } & Stem & Spathulenol (20.0 \%) & [107] \\
\hline & & B-Bisabolol (8.6 \%) & \\
\hline & & Caryophyllene oxide (8.0 \%) & \\
\hline & Fruit & Y-Terpinene (4.5\%) & \\
\hline & & a-Muurolene (3.9\%) & \\
\hline \multirow[t]{3}{*}{ Eryngium rosulatum P. W. Michael ined. } & Aerial parts & $\beta$-Elemene (16.0\%) & [118] \\
\hline & & Bicyclogermacrene (12.5 \%) & \\
\hline & & 8-Elemene (7.0 \%) & \\
\hline
\end{tabular}


Table 3 The major components in the essential oils of various Eryngium species (Continued)

\begin{tabular}{|c|c|c|c|}
\hline \multirow[t]{2}{*}{ Eryngium thorifolium Boiss. } & \multirow[t]{2}{*}{ Flowering aerial parts } & (1R)-a-Pinene (58.6 \%) & \multirow[t]{2}{*}{ [37] } \\
\hline & & Limonene (3.14 \%) & \\
\hline \multirow[t]{2}{*}{ Eryngium tricuspidatum L. } & \multirow[t]{2}{*}{ Aerial parts } & a-Bisabolol (32.6 \%) & \multirow[t]{2}{*}{ [34] } \\
\hline & & a-Curcumene (6.5\%) & \\
\hline \multirow[t]{7}{*}{ Eryngium vesiculaosum Labill. } & \multirow[t]{3}{*}{ Winter leaves } & $\beta$-Caryophyllene (20.3\%) & \multirow[t]{7}{*}{119} \\
\hline & & Germacrene D (19.2 \%) & \\
\hline & & a-Humulene (8.8\%) & \\
\hline & \multirow[t]{4}{*}{ Summer leaves } & Bicyclogermacrene (22.2\%) & \\
\hline & & $\beta$-Caryophyllene (15.6\%) & \\
\hline & & Germacrene D (15.8\%) & \\
\hline & & a-Humulene (8.1\%) & \\
\hline \multirow[t]{14}{*}{ Eryngium yuccifolium Michaux. } & \multirow[t]{6}{*}{ Leaves } & Germacrene D (18.3 \%) & \multirow[t]{14}{*}{120} \\
\hline & & Terpinolene (17.8\%) & \\
\hline & & Bicyclogermacrene (8.8 \%) & \\
\hline & & a-Pinene (7.6 \%) & \\
\hline & & $\beta$-Caryophyllene (6.2\%) & \\
\hline & & Falcarinol (9.6\%) & \\
\hline & \multirow[t]{5}{*}{ Stalks } & Germacrene D (38.4 \%) & \\
\hline & & ү-Amorphene (12.2\%) & \\
\hline & & Bicyclogermacrene (10.1\%) & \\
\hline & & Bicyclosesquiphellandrene (3.4\%) & \\
\hline & & Falcarinol (3.2 \%) & \\
\hline & \multirow[t]{3}{*}{ Roots } & Terpinolene (25.8\%) & \\
\hline & & Trans- $\beta$-Bergamotene (18.6\%) & \\
\hline & & Benzaldehyde 2,3,6-trimethylbenzaldehyde (13.9\%) & \\
\hline
\end{tabular}

Trichophyton mentagrophytes FF7, Epidermophyton floccosum FF9, M. gypseum CECT 2905, T. rubrum CECT 2794, T. mentagrophytes var interdigitale CECT 2958 and T. verrucosum CECT 2992), five Candida species (two clinical isolates from recurrent cases of vulvovaginal or oral candidosis, C. krusei (H9) and C. guillermondii MAT23, along with three reference species of C. albicans ATCC 10231, C. tropicalis ATCC 13803, and C. parapsilosis ATCC 90018) and a strain of Cryptococcus neoformans CECT 1078. Using the macrodilution broth method the MIC and Minimal Lethal Concentrations (MLC) were determined. The results disclosed that the essential oil did not possess antifungal activity against Candida strains, with the exception of C. guillermondii, which resulted to be sensitive to the antifungal activity of the oil with a MIC and MLC of $2.5 \mu \mathrm{L} / \mathrm{mL}$. On the contrary, the essential oil had MIC values of $0.16-0.32 \mu \mathrm{L} / \mathrm{mL}$ against all the tested dermatophyte species. The essential oil was further studied to determine its chemical composition by GC-MS and the authors suggested that caryophyllene derived compounds [isocaryophyllen-14- al (16.2\%), 14-hidroxy- $\beta$-caryophyllene (13.4 \%), caryophyllene oxide $(7.6 \%)$ and $E$ - $\beta$-caryophyllene $(6.3 \%)]$ are probably the responsible for the antifungal activity [97].

Finally, in 2013, the antiphytoviral activity of the essential oils obtained by water distillation of the aerial parts of E. alpinum and E. amethystinum cultivated in Croatia was described. The antiviral activity was shown in Chenopodium quinoa treated with the essential oils (250 ppm) prior to the inoculation of cucumber mosaic virus associated with a satellite RNA. The number of leaf local lesions, registered in the presence of the essential oils, was strongly reduced from an average value of $14.9 \pm 0.8$ to $3.3 \pm 0.2$ and $2.9 \pm 0.2$, for $E$. alpinum and E. amethystinum essential oils, respectively. The authors ascribed the antiviral activity to the occurrence of caryophyllene oxide and $\beta$-caryophyllene, which were identified as the major components of $E$. alpinum and E. amethystinum, respectively. Moreover, other components such as germacrene D, $\alpha$-bisabolol, and $\gamma$-eudesmol, which were detected as minor oil 
Table 4 Antimicrobial activity of Eryngium species extracts

\begin{tabular}{|c|c|c|c|c|c|}
\hline $\begin{array}{l}\text { Eryngium } \\
\text { plant }\end{array}$ & Type of extract & Used method & Microbes & $\begin{array}{l}\text { Positive } \\
\text { control }\end{array}$ & Ref. \\
\hline E. foetidum & $\begin{array}{l}\text { Methanolic extract } \\
\text { of leaves }\end{array}$ & disk diffusion technique & 6 clinical strains of Helicobacter pylori & $n s^{a}$ & [92] \\
\hline E. foetidum & $\begin{array}{l}\text { hexane, dichloromethane, } \\
\text { and methanol extracts }\end{array}$ & disk diffusion technique & Plasmodium falciparum & chloroquine & [93] \\
\hline E. maritimum & $\begin{array}{l}\text { hydrodistillation of the } \\
\text { aerial parts }\end{array}$ & $\begin{array}{l}\text { agar diffusion method } \\
\text { and minimum inhibitory } \\
\text { concentration (MIC) }\end{array}$ & L. monocytogenes E. coli. & $n s^{\mathrm{a}}$ & [94] \\
\hline E. maritimum & $\begin{array}{l}\text { hydromethanolic extract } \\
\text { of leaves }\end{array}$ & microdilution method & $\begin{array}{l}\text { S. aureus subsp. aureus, Micrococcus luteus, } \\
\text { B. cereus. S. enterica subsp. arizonae } \\
\text { S. enterica subsp. Montevideo P. aeruginosa, } \\
\text { P. fluorescens, P. marginalis E. coli Erwinia } \\
\text { carotovora subsp. carotovora, Candida albicans }\end{array}$ & $n s^{a}$ & [95] \\
\hline $\begin{array}{l}\text { E. creticum, E. } \\
\text { campestre, } E \text {. } \\
\text { thorifolium }\end{array}$ & $\begin{array}{l}\text { essential oils from aerial } \\
\text { and root parts }\end{array}$ & disk diffusion method & $\begin{array}{l}9 \text { clinical strains of methicillin-resistant } \\
\text { S. aureus (MRSA) }\end{array}$ & $n s^{a}$ & [37] \\
\hline E. duriaei & $\begin{array}{l}\text { essential oil obtained by } \\
\text { water distillation of the } \\
\text { aerial parts }\end{array}$ & macrodilution broth method & $\begin{array}{l}\text { Microsporum canis FF1, Trichophyton mentagrophytes } \\
\text { FF7, Epidermophyton floccosum FF9, M. gypseum } \\
\text { CECT 2905, T. rubrum CECT 2794, T. mentagrophytes } \\
\text { var interdigitale CECT 2958 T. verrucosum CECT } 2992 \\
\text { Candida. guillermondii MAT23, Cryptococcus neoformans } \\
\text { CECT 1078. }\end{array}$ & $\mathrm{ns}^{\mathrm{a}}$ & [97] \\
\hline $\begin{array}{l}\text { E. alpinum, } E \text {. } \\
\text { amethystinum }\end{array}$ & $\begin{array}{l}\text { essential oils obtained by } \\
\text { water distillation of the } \\
\text { aerial parts }\end{array}$ & $\begin{array}{l}\text { count of leaf local lesions in } \\
\text { Chenopodium quinoa treated } \\
\text { with the essential oils prior to } \\
\text { the inoculation of virus }\end{array}$ & cucumber mosaic virus associated with a satellite RNA & $n s^{a}$ & [98] \\
\hline
\end{tabular}

${ }^{a}$ not specified

constituents, might be responsible for the inhibition of viral infection [98].

The summary of the literature data was reported in Table 4.

\section{Conclusion}

The present paper shows that essential oils and extracts obtained from various Eryngium species have broad range antimicrobial activity against several strains of gram-positive and gram-negative bacteria, some species of fungi and yeasts, and viruses. The findings point out to the fact that these activities can be ascribed to the presence of different phytochemicals, especially apolar compounds. However, our search at https://clinicaltrials.gov/ with keyword "Eryngium" accessed on February 9, 2015 showed no clinical trial regarding the beneficial effects of the genus Eryngium plants against different types of infection available up to date. In addition, there are only a few papers on the toxicity of the active constituents belonging to the genus Eryngium plants, which resulted to be cytotoxic especially at high doses. It can be suggested that more toxicity studies should be carried out prior to the clinical trials.

According to this study, we conclude that the essential oils and extracts of those Eryngium species that have been submitted to in vitro investigation (Table 4) should be proceeded to toxicological studies and in vivo experiments as multi-target antimicrobial agents for the treatment of human infectious diseases, especially antibiotic-resistant bacterial infections. Thus, it is not quite possible to make a clear statement or comment about their clinical uses. Hence, we recommend that future studies should be performed on:

- toxicity of the Eryngium plant extracts and essential oil resulted to be active in in vitro experiments.

- clinical studies of the safe extracts of Eryngium species with in vitro activity,

- finding the exact mechanism underlying the antibacterial effects of the essential oils and extracts of members of the genus Eryngium and their antibacterial constituents,

- separation, isolation, and structure identification of the most antibacterial constituents of the essential oils and extracts of different members of the genus Eryngium and their interactions with foods as well as common synthetic antibacterial compounds, - ascertaining the most effective and safe doses for clinical studies regarding the antibacterial effects of the essential oils and extracts of different genus Eryngium species against different infectious diseases.

Competing interests

The authors declare that they have no competing interests. 


\section{Authors' contributions}

SMN and IEO designed the paper, SAE, MI and MD collected and selected and analyzed the literature data, SAE, SFN, SMN, IEO and MD wrote the paper. All authors participated in the analysis and interpretation of literature data, corrected the paper and approved the final manuscript.

\section{Acknowledgement}

Declared none.

\section{Author details}

'Department of Pharmacognosy, Faculty of Pharmacy, Ankara University, 06100 Ankara, Turkey. ${ }^{2}$ Applied Biotechnology Research Center, Baqiyatallah University of Medical Sciences, P.O. Box 19395-5487, Tehran, Iran.

${ }^{3}$ Department of Pharmacognosy, Faculty of Pharmacy, Gazi University, 06330 Ankara, Turkey. ${ }^{4}$ Department of Drug Sciences, Medicinal Chemistry and Pharmaceutical Technology Section, University of Pavia, Pavia, Italy. ${ }^{5}$ Health Research Center, Baqiyatallah University of Medical Sciences, Tehran, Iran.

\section{Received: 23 October 2015 Accepted: 3 December 2015}

\section{Published online: 14 December 2015}

\section{References}

1. World Health Organization. World health statistics 2010. WHO Press. Geneva, Switzerland. 2010.

2. Nabavi SM, Marchese A, Izadi M, Curti V, Daglia M, Nabavi SF. Plants belonging to the genus Thymus as antibacterial agents: From farm to pharmacy. Food Chem. 2015;173:339-47.

3. Högberg LD, Heddini A, Cars $O$. The global need for effective antibiotics: challenges and recent advances. Trends Pharmacol Sci. 2010;31(11):509-15.

4. Alinezhad H, Azimi R, Zare M, Ebrahimzadeh MA, Eslami S, Nabavi SF, et al. Antioxidant and antihemolytic activities of ethanolic extract of flowers, leaves, and stems of Hyssopus officinalis L. Var. angustifolius. Int J Food Prop. 2013;16(5):1169-78.

5. Nabavi SF, Nabavi SM, Habtemariam S, Moghaddam AH, Sureda A, Jafari M, et al. Hepatoprotective effect of gallic acid isolated from Peltiphyllum peltatum against sodium fluoride-induced oxidative stress. Ind Crops Prod. 2013;44:50-5.

6. Curti V, Capelli E, Boschi F, Nabavi SF, Bongiorno Al, Habtemariam S, et al. Modulation of human miR-17-3p expression by methyl 3-0-methyl gallate as explanation of its in vivo protective activities. Mol Nutr Food Res. 2014; 58(9):1776-84

7. Nabavi SF, Daglia M, Moghaddam AH, Habtemariam S, Nabavi SM Curcumin and liver disease: from chemistry to medicine. Compr Rev Food Sci Food Saf. 2014;13(1):62-77.

8. Nabavi SF, Russo GL, Daglia M, Nabavi SM. Role of quercetin as an alternative for obesity treatment: you are what you eat! Food Chem. 2015; 179:305-10.

9. Nabavi SF, Nabavi SM, Setzer NW, Nabavi SA, Nabavi SA, Ebrahimzadeh MA. Antioxidant and antihemolytic activity of lipid-soluble bioactive substances in avocado fruits. Fruits. 2013:68(03):185-93.

10. Daglia M. Polyphenols as antimicrobial agents. Curr Opin Biotechnol. 2012; 23(2):174-81.

11. Simoes M, Bennett RN, Rosa EA. Understanding antimicrobial activities of phytochemicals against multidrug resistant bacteria and biofilms. Nat Prod Rep. 2009;26(6):746-57

12. Cowan MM. Plant products as antimicrobial agents. Clin Microbiol Rev. 1999;12(4):564-82

13. Palá-Paúl J, Perez-Alonso MJ, Velasco-Negueruela A, Vadaré J, Villa AM, Sanz J, et al. Essential oil composition of the different parts of Eryngium bourgatii Gouan from Spain. J Chromatogr A. 2005;1074(1):235-9.

14. Capetanos C, Saroglou V, Marin PD, Simić A, Skaltsa HD. Essential oil analysis of two endemic Eryngium species from Serbia. J Serb Chem Soc. 2007; 72(10):961-5.

15. Wörz A, Diekmann H. Classification and evolution of the genus Eryngium L. (Apiaceae-Saniculoideae): results of fruit anatomical and petal morphological studies. Plant Divers Evol. 2010;128(3-4):387-408.

16. Wörz A. A new subgeneric classification of the genus Eryngium L. (Apiaceae, Saniculoideae). Bot Jahrbücher. 2005;126(2):253-9.

17. Calvino Cl, Martínez SG, Downie SR. The evolutionary history of Eryngium (Apiaceae, Saniculoideae): Rapid radiations, long distance dispersals, and hybridizations. Mol Phylogenet Evol. 2008;46(3):1129-50.
18. Calviño Cl, Downie SR. Circumscription and phylogeny of Apiaceae subfamily Saniculoideae based on chloroplast DNA sequences. Mol Phylogenet Evol. 2007;44(1):175-91.

19. Magee AR, van Wyk B-E, Tilney PM, van der Bank M. A Taxonomic Revision Of the South African Endemic Genus Arctopus (Apiaceae, Saniculoideae) 1. Ann Missouri Bot Garden. 2008:95(3):471-86.

20. Singh S, Singh D, Banu S, Salim K. Determination of bioactives and antioxidant activity in Eryngium foetidum L.: a traditional culinary and medicinal herb. Proc Natl Acad Sci, India Section B: Biological Sciences. 2013;83(3):453-60

21. Ignacimuthu S, Arockiasamy S, Antonysamy M, Ravichandran P. Plant regeneration through somatic embryogenesis from mature leaf explants of Eryngium foetidum, a condiment. Plant Cell Tissue Organ Cult. 1999;56(2):131-7.

22. Shavandi MA, Haddadian Z, Ismail MHS. Eryngium foetidum L. Coriandrum sativum and Persicaria odorata L.: A review. J Asian Sci Res. 2012;2(8):410-26.

23. Singh B, Ramakrishna Y, Ngachan S. Spiny coriander (Eryngium foetidum L.): a commonly used, neglected spicing-culinary herb of Mizoram, India. Genet Resour Crop Evol. 2014;61(6):1085-90.

24. Paul J, Seaforth C, Tikasingh T. Eryngium foetidum L.: a review. Fitoterapia. 2011;82(3):302-8.

25. Kupeli E, Kartal M, Aslan S, Yesilada E. Comparative evaluation of the antiinflammatory and antinociceptive activity of Turkish Eryngium species. J Ethnopharmacol. 2006;107(1):32-7.

26. De Natale A, Pollio A. Plants species in the folk medicine of Montecorvino Rovella (inland Campania, Italy). J Ethnopharmacol. 2007;109(2):295-303.

27. Wang P, Su Z, Yuan W, Deng G, Li S. Phytochemical constituents and pharmacological activities of Eryngium L. (Apiaceae). Pharm Crops. 2012;3:99-120.

28. Nabavi S, Nabavi S, Alinezhad H, Zare M, Azimi R. Biological activities of flavonoid-rich fraction of Eryngium caucasicum Trautv. Eur Rev Med Pharmacol Sci. 2012;16:81-7.

29. Eslami S, Ebrahimzadeh M, Moghaddam HA, Nabavi S, Jafari N, Nabavi S Renoprotective effect of Eryngium caucasicum in gentamicin-induced nephrotoxic mice. Arch Biol Sci. 2011;63(1):157-60.

30. Anam EM. A novel triterpenoid saponin from Eryngium foetidum. Indian J Chem Section B. 2002;41(7):1500-3.

31. Khoshbakht K, Hammer K, Pistrick K. Eryngium caucasicum Trautv. cultivated as a vegetable in the Elburz Mountains (Northern Iran). Genet Resour Crop Evol. 2007:54(2):445-8.

32. Homer S, Baccus-Taylor GS, Akingbala JA, Hutchinson SD, editors. Antibacterial efficacy of Eryngium foetidum (culantro) against select foodborne pathogens: Proceedings of the West Indies Agricultural Economics Conference. 2009.

33. Türker $H$, Yildirim AB, Karakaş FP, Köylüoglu H. Antibacterial activities of extracts from some Turkish endemic plants on common fish pathogens. Turk J Biol. 2009;33(1):73-8.

34. Merghache D, Boucherit-Otmani Z, Merghache S, Chikhi I, Selles C, Boucherit K. Chemical composition, antibacterial, antifungal and antioxidant activities of Algerian Eryngium tricuspidatum L. essential oil. Nat Prod Res. 2014;28(11):795-807.

35. Dehghanzadeh N, Ketabchi S, Alizadeh A. Essential oil composition and antibacterial activity of Eryngium caeruleum grown wild in Iran. J Essential Oil Bearing Plants. 2014;17(3):486-92.

36. Khalfallah A, Berrehal D, Kabouche A, Karioti A, Bilia A-R, Kabouche Z. Flavonoids, antioxidant and antibacterial activities of Eryngium triquetrum. Chem Nat Comp. 2014;50(1):130-2.

37. Çelik A, Aydınlık N, Arslan I. Phytochemical constituents and inhibitory activity towards methicillin-resistant Staphylococcus aureus strains of Eryngium species (Apiaceae). Chem Biodivers. 2011;8(3):454-9.

38. Ozcelik B, Kusmenoglu S, Turkoz S, Abbasoglu U. Antimicrobial activities of plants from the Apicaceae. Pharm Biology. 2004;42(7):526-8.

39. Sefidkon F, Dabiri M, Alamshahi A. Chemical composition of the essential oil of Eryngium billardieri F. Delaroche from Iran. J Essential Oil Res. 2004;16(1): 42-3.

40. Ebrahimzadeh M, Nabavi S, Nabavi S. Antioxidant activity of leaves and inflorescence of Eryngium caucasicum Trautv at flowering stage. Pharmacogn Res. 2009;1(6):435

41. Aburjai T, Hudaib M, Tayyem R, Yousef M, Qishawi M. Ethnopharmacological survey of medicinal herbs in Jordan, the Ajloun Heights region. J Ethnopharmacol. 2007;110(2):294-304. 
42. Goleniowski ME, Bongiovanni G, Palacio L, Nuñez C, Cantero J. Medicinal plants from the "Sierra de Comechingones", Argentina. J Ethnopharmacol. 2006;107(3):324-41.

43. Redzic S. Wild medicinal plants and their usage in traditional human therapy (Southern Bosnia and Herzegovina, W. Balkan). J Med Plants Res. 2010;4(11):1003-27.

44. Morton JF. Some folk-medicine plants of Central American markets. Pharm Biol. 1977;15(4):165-92.

45. Morton JF. Caribbean and Latin American folk medicine and its influence in the United States. Pharm Biol. 1980;18(2):57-75.

46. Mathias ME. Magic, myth and medicine. Econ Bot. 1994;48(1):3-7.

47. Wang P, Yuan W, Deng G, Su Z, Li S. Triterpenoid saponins from Eryngium yuccifolium 'Kershaw Blue'. Phytochem Lett. 2013;6(2):306-9.

48. de la Luz C-GM, Fernández-Arroyo S, Joven J, Segura-Carretero A. Comprehensive characterization by UHPLC-ESI-Q-TOF-MS from an Eryngium bourgatii extract and their antioxidant and anti-inflammatory activities. Food Res Int. 2013;50(1):197-204.

49. Erdem SA, Arıhan O, Offer AM, Iskit A, Miyamoto T, Kartal M, et al. Antinociceptive activity of Eryngium kotschyi Boiss. root extracts. Planta Med. 2011;77(12):F66.

50. Ozarowski M, Thiem B, Mikolajczak PL, Piasecka A, Kachlicki P, Szulc M, et al. Improvement in Long-Term Memory following Chronic Administration of Eryngium planum Root Extract in Scopolamine Model: Behavioral and Molecular Study. Evid Based Complement Alternat Med. 2015;2015:145140.

51. Khalili M, Dehdar T, Hamedi F, Ebrahimzadeh M, Karami M. Antihypoxic activities of Eryngium caucasicum. Eur Rev Med Pharmacol Sci. 2015;19(17):3282-5.

52. Dawilai S, Muangnoi C, Praengamthanachoti P, Tuntipopipat S. Antiinflammatory activity of bioaccessible fraction from Eryngium foetidum leaves. BioMed Res Int. 2013;2013:958567.

53. Mozumder S, Hossain M. Effect of seed treatment and soaking duration on germination of Eryngium foetidum L. seeds. Int J Hort. 2013;3:1046-51.

54. Njenga J. Production of Eryngium. NC Flower Growers' Bulletin. 1995;40:9-11.

55. Martin K. In vitro propagation of the herbal spice Eryngium foetidum L. on sucrose-added and sucrose-free medium without growth regulators and $\mathrm{CO}_{2}$ enrichment. Sci Hort. 2004;102(2):277-82.

56. Erdelmeier $\mathrm{C}$, Sticher O. Coumarin derivatives from Eryngium campestre. Planta Med. 1985;51(5):407-9.

57. Erdelmeier CA, Sticher O. A cyclohexenone and a cyclohexadienone glycoside from Eryngium campestre. Phytochemistry. 1986;25(3):741-3.

58. Hohmann J, Pall Z, Günther G, Mathe I. Flavonolacyl glycosides of the aerial parts of Eryngium campestre. Planta Med. 1997;63(1):96.

59. Kartal M, Mitaine-Offer A-C, Abu-Asaker M, Miyamoto T, Calis I, Wagner H, et al. Two new triterpene saponins from Eryngium campestre. Chem Pharm Bull. 2005;53(10):1318-20

60. Dalar A, Türker M, Zabaras D, Konczak I. Phenolic composition, antioxidant and enzyme inhibitory activities of Eryngium bornmuelleri leaf. Plant Foods Hum Nutr. 2014;69(1):30-6.

61. Hiller K, Keipert M, Pfeifer S, Kraft R. The leaf sapogenin spectrum in Eryngium planum L. 20. Contribution on the content of several Saniculoideae. Pharmazie. 1974;29(1):54

62. Hiller K, Nguyen K, Franke P. Isolation of 3-OD-glucopyranosyl oleanolic acid 28-OD-xylo-pyranoside from Eryngium bromeliifolium Delar. 29. Constituents of some Saniculoideae. Pharmazie. 1978;33(1):78-80.

63. Kartal M, Mitaine-Offer A-C, Paululat T, Abu-Asaker M, Wagner H, Mirjolet J-F, et al. Triterpene saponins from Eryngium campestre. J Nat Prod. 2006;69(7):1105-8.

64. Zhang Z, Li S, Ownby S, Wang P, Yuan W, Zhang W, et al. Phenolic compounds and rare polyhydroxylated triterpenoid saponins from Eryngium yuccifolium. Phytochemistry. 2008;69(10):2070-80

65. Erdem SA, Mitaine-Offer AC, Miyamoto T, Kartal M, Lacaille-Dubois MA Triterpene saponins from Eryngium kotschyi. Phytochemistry. 2015;110:160-5.

66. Hiller K, Thi N, Franke P. On saponin of Eryngium bromeliifoloium Delar. 19. Information on the components of various Saniculoideae. Pharmazie. 1973; 28(8):546.

67. Hiller K, Thi N, Döhnert H, Franke P. Isolation of new ester sapogenins from Eryngium giganteum MB 22. Knowledge of various Saniculoideae components. Pharmazie. 1975;30(2):105.

68. Hiller K, Nguyen K, Döhnert H, Franke P. The saponine-saponinogen spectrum in Eryngium amethystinum L. 27. Contribution to chemicals contained in various Saniculoidae. Pharmazie. 1977;32(3):184.

69. Hiller K, Keipert M, Pfeifer S, Tökes L, Maddox M. Structure of Erygium sapogenins. 14. Information on components of some Saniculoideae. Pharmazie. 1970;25(12):769.
70. Hiller K, Keipert M, Pfeifer S, Tokes L, Nelson J. Erynginol A- A new triterpenesapogenin. 18. Knowledge of component substances of some saniculoideae. Pharmazie 1973;28(6):409-10.

71. Hiller K, Von Thi N, Lehmann G, Gründemann E. Betulinic acid-a sapogenin in Eryngium bromeliifolium Delar. 21. The contents of a Saniculoidea. Pharmazie. 1974;29(2):148.

72. Hiller K, Keipert M, Missbach U, Lehmann G. Eryngiumgenin E-a new ester sapogenin. 23. The contents of some Saniculoideae. Pharmazie. 1975;30(5):336

73. Hiller K, Nguyen K, Franke P, Hintsche R. Isolation of betulic acid 3-O-beta-D glucoside, a saponin of Eryngium bromeliifolium Delar. 26. The contents of Saniculoidease. Pharmazie. 1976;31(12):891.

74. Hiller K, Von Mach B, Franke P. Saponins of Eryngium maritimum L. 25. Contents of various Saniculoideae. Pharmazie. 1976;31(1):53.

75. Voigt G, Thiel P, Hiller K, Franke P, Habisch D. Zur struktur des hauptsaponins der Wurzeln von Eryngium planum L.. XL: Zur Kenntnis der inhaltsstoffe einiger Saniculoideae. Pharmazie 1985;40(9):656-59.

76. Kowalczyk M, Masullo M, Thiem B, Piacente S, Stochmal A, Oleszek W. Three new triterpene saponins from roots of Eryngium planum. Nat Prod Res. 2014;28(9):653-60.

77. Aslan S, Kartal M. GC-MS Analysis of Eryngium maritumum L. volatile oil. Planta Med 2006;72(11):P_340.

78. Hiller K, Otto A, Grundemann E. Isolation of kaempferol-3-o-(6-O-beta-Dglucopyranosyl)-beta-D-galactopyranoside, a new flavonol glycoside from Eryngium planum I. 34. on the knowledge of the constituents of some saniculoideae. Pharmazie. 1980;35(2):113-4.

79. Kartnig T, Wolf J. Flavonoids from the aboveground parts of Eryngium campestre. Planta Med. 1993;59(3):285.

80. Hawas UW, El-Kassem T, Lamia A, Awad M, Hanem AA, Taie H. Anti-Alzheimer, antioxidant activities and flavonol glycosides of Eryngium campestre L. Curr Chem Biol. 2013;7(2):188-95.

81. Pinar M, Galan MP. Coumarins from Eryngium ilicifolium. J Nat Prod. 1985; 48(5):853-4.

82. Le Claire E, Schwaiger S, Banaigs B, Stuppner H, Gafner F. Distribution of a new rosmarinic acid derivative in Eryngium alpinum L. and other Apiaceae. J. Agric Food Chem. 2005;53(11):4367-72.

83. Marčetić MD, Petrović SD, Milenković MT, Niketić MS. Composition, antimicrobial and antioxidant activity of the extracts of Eryngium palmatum Pančić and Vis. (Apiaceae). Cent Eur J Biol. 2014;9(2):149-55.

84. Drake D, Lam J. Seseli acetylene from Eryngium bourgatti. Phytochemistry. 1972;11(8):2651-2

85. Lam J, Christensen LP, Thomasen T. Acetylenes from roots of Eryngium bourgatii. Phytochemistry. 1992;31(8):2881-2.

86. Ayoub N, Kubeczka K-H, Nawwar M. An unique n-propyl sesquiterpene from Eryngium creticum L. (Apiaceae). Pharmazie. 2003;58(9):674-6.

87. Korbel E, Bighelli A, Kurowska A, Kalemba D, Casanova J. New cis-chrysanthenyl esters from Eryngium planum L. Nat Prod Commun. 2008;3(2):113-6.

88. Garcia M, Saenz M, Gomez M, Fernandez M. Topical anti-inflammatory activity of phytosterols isolated from Eryngium foetidum on chronic and acute inflammation models. Phytother Res. 1999;13(1):78-80.

89. Muckensturm B, Boulanger A, Farahi M, Reduron J. Secondary metabolites from Eryngium species. Nat Prod Res. 2010;24(5):391-7.

90. Forbes W, Gallimore W, Mansingh A, Reese P, Robinson R. Eryngial (trans-2dodecenal), a bioactive compound from Eryngium foetidum: its identification, chemical isolation, characterization and comparison with ivermectin in vitro. Parasitology. 2014;141(02):269-78.

91. Alzoreky N, Nakahara K. Antibacterial activity of extracts from some edible plants commonly consumed in Asia. Int J Food Microbiol. 2003;80(3):223-30.

92. Ndip RN, Tarkang AEM, Mbullah SM, Luma HN, Malongue A, Ndip LM, et al. In vitro anti-Helicobacter pylori activity of extracts of selected medicinal plants from North West Cameroon. J Ethnopharmacol. 2007;114(3):452-7.

93. Roumy V, Garcia-Pizango G, Gutierrez-Choquevilca A-L, Ruiz L, Jullian V, Winterton P, et al. Amazonian plants from Peru used by Quechua and Mestizo to treat malaria with evaluation of their activity. J Ethnopharmacol. 2007;112(3):482-9.

94. Darriet F, Bendahou M, Desjobert J-M, Costa J, Muselli A. Bicyclo [4.4.0] decane oxygenated sesquiterpenes from Eryngium maritimum essential oil. Planta Med. 2012;78(4):386

95. Meot-Duros L, Le Floch G, Magné C. Radical scavenging, antioxidant and antimicrobial activities of halophytic species. J Ethnopharmacol. 2008;116(2): 258-62.

96. Baytop T. Therapy with Medicinal Plants in Turkey (Past and Present). 1999;2:169. 
97. Abou-Jawdah Y, Sobh H, Salameh A. Antimycotic activities of selected plant flora, growing wild in Lebanon, against phytopathogenic fungi. J Agric Food Chem. 2002;50(11):3208-13.

98. Dunkić V, Vuko E, Bezić N, Kremer D, Ruščić M. Composition and antiviral activity of the essential oils of Eryngium alpinum and E. amethystinum. Chem Biodivers. 2013;10(10):1894-902.

99. Flamini G, Tebano M, Cioni PL. Composition of the essential oils from leafy parts of the shoots, flowers and fruits of Eryngium amethystinum from Amiata Mount (Tuscany, Italy). Food Chem. 2008;107(2):671-4.

100. Morteza-Semnani K. Essential oil composition of Eryngium bungei Boiss. J Essential Oil Res. 2005;17(5):485-6.

101. Mohammadhosseini M, Mahdavi B, Akhlaghi H. Characterization and chemical composition of the volatile oils from aerial parts of Eryngium bungei Bioss. (Apiaceae) by using traditional hydrodistillation, microwave assisted hydrodistillation and head space solid phase microextraction methods prior to GC and GC/MS analyses: A comparative approach. J Essential Oil Bearing Plants. 2013;16(5):613-23.

102. Assadian F, Masoudi S, Nematollahi F, Rustaiyan A, Larijani K, Mazloomifar H. Volatile constituents of Xanthogalum purpurascens Ave-Lall., Eryngium caeruleum MB and Pimpinella aurea DC. Three Umbelliferae herbs growing in Iran. J Essential Oil Res. 2005;17(3):243-5.

103. Pala-Paul J, Usano-Alemany J, Soria AC, Perez-Alonso MJ, Brophy JJ. Essential oil composition of Eryngium campestre L. growing in different soil types. A preliminary study. Nat Prod Commun. 2008;3(7):1121-6.

104. Hashemabadi D, Kaviani B. Chemical constituents of essential oils extracted from the leaves and stems of Eryngium caucasicum Trautv. from Iran. J Essential Oil Bearing Plants. 2011;14(6):693-8.

105. Palá-Paúl J, Brophy JJ, Pérez-Alonso MJ, Usano J, Soria SC. Essential oil composition of the different parts of Eryngium corniculatum Lam. (Apiaceae) from Spain. J Chromatogr A. 2007;1175(2):289-93.

106. Cavaleiro C, Gonçalves MJ, Serra D, Santoro G, Tomi F, Bighelli A, et al. Composition of a volatile extract of Eryngium duriaei subsp. juresianum (M. Laínz) M. Laínz, signalised by the antifungal activity. J Pharm Biomed Anal. 2011; 54(3):619-22.

107. Brophy JJ, Goldsack RJ, Copeland LM, Palá-Paúl J. Essential oil of Eryngium L. species from New South Wales (Australia). J Essential Oil Res. 2003;15(6): 392-7.

108. Martins AP, Salgueiro LR, da Cunha AP, Vila R, Cañigueral S, Tomi F, et al. Essential oil composition of Eryngium foetidum from S. Tome e Principe. J Essential Oil Res. 2003;15(2):93-5.

109. Cardozo E, Rubio M, Rojas L, Usubillaga A. Composition of the essential oil from the leaves of Eryngium foetidum L. from the Venezuelan Andes. J Essential Oil Res. 2004;16(1):33-4.

110. Thi NDT, Anh TH, Thach LN. The essential oil composition of Eryngium foetidum $L$ in South Vietnam extracted by hydrodistillation under conventional heating and microwave irradiation. J Essential Oil Bearing Plants. 2008;11(2):154-61.

111. Pino JA, Rosado A, Fuentes V. Composition of the leaf oil of Eryngium foetidum L. from Cuba. J Essential Oil Res. 1997;9(4):467-8.

112. Palá-Paúl J, Pérez-Alonso MJ, Velasco-Negueruela A, Varadé J, Villa AM, Sanz J, et al. Analysis of the essential oil composition from the different parts of Enyngium glaciale Boiss. from Spain. J Chromatogr A. 2005;1094(1):179-82.

113. Maggio A, Bruno M, Formisano C, Rigano D, Senatore F. Chemical composition of the essential oils of three species of Apiaceae growing wild in Sicily: Bonannia graeca, Eryngium maritimum and Opopanax chironium. Nat Prod Commun. 2013;8(6):841-4

114. Darriet F, Andreani S, De Cian MC, Costa J, Muselli A. Chemical variability and antioxidant activity of Eryngium maritimum L. essential oils from Corsica and Sardinia. Flav Fragr J. 2014;29(1):3-13.

115. Marcetic M, Petrovic S, Milenkovic M, Vujisic L, Tesevic V, Niketic M. Composition and antimicrobial activity of root essential oil of Balkan endemic species Eryngium palmatum. Chem Nat Comp. 2014;49(6):1140-2

116. Cobos MI, Rodriguez JL, De Petre A, Spahn E, Casermeiro J, Lopez AG, et al. Composition of the essential oil of Eryngium paniculatum Cav. J Essential Oil Res. 2002;14(2):82-3.

117. Thiem B, Kikowska M, Kurowska A, Kalemba D. Essential oil composition of the different parts and in vitro shoot culture of Eryngium planum L. Molecules. 2011;16(8):7115-24.

118. Palá-Paúl J, Copeland LM, Brophy JJ, Goldsack RJ. Essential oil composition of Eryngium rosulatum PW Michael ined.: A new undescribed species from eastern Australia. Biochem System Ecol. 2006:34(11):796-801.
119. Palá-Paúl J, Brophy JJ, Goldsack RJ, Copeland LM, Pérez-Alonso MJ, VelascoNegueruela A. Essential oil composition of the seasonal heterophyllous leaves of Eryngium vesiculosum from Australia. Austr J Bot. 2003;51(5):497-501.

120. Ayoub N, Al-Azizi M, König W, Kubeczka KH. Essential oils and a novel polyacetylene from Eryngium yuccifolium Michaux. (Apiaceae). Flav Fragr J. 2006;21(6):864-8.

\section{Submit your next manuscript to BioMed Central and we will help you at every step:}

- We accept pre-submission inquiries

- Our selector tool helps you to find the most relevant journal

- We provide round the clock customer support

- Convenient online submission

- Thorough peer review

- Inclusion in PubMed and all major indexing services

- Maximum visibility for your research

Submit your manuscript at www.biomedcentral.com/submit
) Biomed Central 